\title{
ATP-binding cassette A1 deficiency causes cardiolipin-driven mitochondrial dysfunction in podocytes
}

\author{
G. Michelle Ducasa, ${ }^{1}$ Alla Mitrofanova, ${ }^{1,2}$ Shamroop K. Mallela, ${ }^{1}$ Xiaochen Liu, ${ }^{1}$ Judith Molina, ${ }^{1}$ Alexis Sloan, ${ }^{1}$ Christopher E. Pedigo, ${ }^{3}$ \\ Mengyuan Ge, Javier Varona Santos, ${ }^{1}$ Yanio Hernandez, ${ }^{1}$ Jin-Ju Kim, ${ }^{1}$ Cyrille Maugeais, ${ }^{4}$ Armando J. Mendez, ${ }^{5}$ Viji Nair, ${ }^{6}$ \\ Matthias Kretzler, ${ }^{6}$ George W. Burke, ${ }^{2}$ Robert G. Nelson, ${ }^{7}$ Yu Ishimoto, ${ }^{8}$ Reiko Inagi, ${ }^{8}$ Santanu Banerjee, ${ }^{2}$ Shaoyi Liu, ${ }^{9}$ \\ Hazel H. Szeto, ${ }^{9}$ Sandra Merscher, ${ }^{1}$ Flavia Fontanesi, ${ }^{10}$ and Alessia Fornoni ${ }^{1}$
}

'Katz Family Division of Nephrology and Hypertension/ Drug Discovery Center, Department of Medicine, University of Miami, Miami, Florida, USA. 2Department of Surgery, University of Miami, Miami, Florida, USA ${ }^{3}$ Department of Internal Medicine, Yale University, New Haven, Connecticut, USA. “'Roche Innovation Center Basel, F. Hoffmann-La Roche Ltd., Basel, Switzerland. ${ }^{5}$ Diabetes Research Institute, University of Miami, Miami, Florida, USA. ${ }^{6}$ Department of Internal Medicine, Division of Nephrology, University of Michigan, Ann Arbor, Michigan, USA. ${ }^{7}$ NIDDK, Phoenix, Arizona, USA. ${ }^{8}$ Division of CKD Pathophysiology, University of Tokyo, Tokyo, Japan. ${ }^{~}$ Social Profit Network Research Lab, Alexandria LaunchLabs, New York, New York, USA. ${ }^{10}$ Department of Biochemistry and Molecular Biology, University of Miami, Miami, Florida, USA.

Fibroblasts from patients with Tangier disease carrying ATP-binding cassette A1 (ABCA1) loss-of-function mutations are characterized by cardiolipin accumulation, a mitochondrial-specific phospholipid. Suppression of ABCA1 expression occurs in glomeruli from patients with diabetic kidney disease (DKD) and in human podocytes exposed to DKD sera collected prior to the development of DKD. We demonstrated that siRNA ABCA1 knockdown in podocytes led to reduced oxygen consumption capabilities associated with alterations in the oxidative phosphorylation (OXPHOS) complexes and with cardiolipin accumulation. Podocyte-specific deletion of Abca1 (Abca1 ${ }^{\mathrm{fl} / \mathrm{fl}}$ ) rendered mice susceptible to DKD, and pharmacological induction of ABCA1 improved established DKD. This was not mediated by free cholesterol, as genetic deletion of sterol-oacyltransferase-1 (SOAT1) in Abca1 ${ }^{\text {fl/fl }}$ mice was sufficient to cause free cholesterol accumulation but did not cause glomerular injury. Instead, cardiolipin mediates ABCA1-dependent susceptibility to podocyte injury, as inhibition of cardiolipin peroxidation with elamipretide improved DKD in vivo and prevented ABCA1-dependent podocyte injury in vitro and in vivo. Collectively, we describe a pathway definitively linking ABCA1 deficiency to cardiolipin-driven mitochondrial dysfunction. We demonstrated that this pathway is relevant to DKD and that ABCA1 inducers or inhibitors of cardiolipin peroxidation may each represent therapeutic strategies for the treatment of established DKD.

\section{Introduction}

Diabetic kidney disease (DKD) remains the most common cause of chronic kidney disease (CKD), comprising about $40 \%$ of patients with CKD (1). However, not all patients with diabetes develop DKD, and aggressive management of blood pressure (2) and glycemia (3) reduces but does not eliminate the risk for DKD, suggesting that factors other than impaired glucose metabolism and altered hemodynamics are involved in the pathogenesis (4).

Conflict of interest: CWB, AF, and SM are inventors on pending or issued patents (US $10,183,038$ and US 10,052,345) aimed at diagnosing or treating proteinuric kidney diseases. They stand to gain royalties from the future commercialization of these patents. AF is Chief Scientific Officer of L\&F Health LLC and is a consultant for Variant Pharmaceuticals. Variant Pharmaceuticals has licensed worldwide rights from L\&F Research to develop and commercialize hydroxypropyl-beta-cyclodextrin for the treatment of kidney disease. AF is Chief Medical Officer of LipoNexT LLC. SM holds equity interest in a company presently commercializing the form of cyclodextrin referenced in this paper. AF and SM are supported by Hoffman-La Roche. AF is supported by Boehringer Ingelheim. Copyright: @ 2019 , American Society for Clinical Investigation.

Submitted: October 11, 2018; Accepted: May 28, 2019; Published: July 22, 2019.

Reference information: J Clin Invest. 2019;129(8):3387-3400.

https://doi.org/10.1172/JCl125316.
Among all affected cellular constituents of the glomerular filtration barrier, podocyte loss correlates with the development of albuminuria in both clinical and experimental DKD (5-7). However, the cause of podocyte loss in DKD remains to be established.

We and others have described glomerular accumulation of lipids in both clinical and experimental DKD (8-11). Of the many genes involved in lipid metabolism, our group has demonstrated decreased ATP-binding cassette A1 (ABCA1) expression in glomerular tissue obtained from research kidney biopsies of Pima Indians with type 2 diabetes (T2D) (9). ABCA1 is a transmembrane protein which, in an ATP-dependent mechanism, regulates the efflux of cholesterol and phospholipids $(12,13)$. Similarly, in a separate T2D Israeli cohort, downregulation of glomerular ABCA1 expression correlated positively with estimated glomerular filtration rate (GFR) (8). While both pharmacological induction of cholesterol efflux with cyclodextrin (9) or genetic overexpression of ABCA1 (14) are sufficient to rescue from DKD or DKD-like glomerulosclerosis, the mechanisms linking ABCA1 deficiency to glomerular injury are unknown. Patients affected by Tangier disease (characterized by ABCA1 loss-of-function mutations) have foamy podocytes on kidney biopsies (15). However, they develop 


\section{Table 1. Enrichment analysis of NP versus $P$ sera-treated podocytes}

\begin{tabular}{|c|c|c|c|c|}
\hline Pathway & Total genes in pathway & Gene count & $P$ value & FDR \\
\hline Signal transduction calcium signaling & 45 & 9 & $1.035 \times 10^{-5}$ & 0.006 \\
\hline Development: leptin signaling via PIJK-dependent pathway & 47 & 8 & 0.0001 & 0.012 \\
\hline Cardiac hypertrophy: NFAT signaling in cardiac hypertrophy & 65 & 9 & 0.0002 & 0.012 \\
\hline
\end{tabular}

only minimal proteinuria, suggesting that ABCA1 deficiency may be sufficient to cause lipid accumulation but not glomerular cell injury (15). In fact, neither ABCA1 siRNA in podocytes in vitro nor podocyte-specific deletion of ABCA1 in vivo is sufficient to cause glomerular injury (14). However, the possibility that ABCA1 deficiency and the related lipid accumulation renders podocytes susceptible to injury in DKD remains to be established.

The observation that fibroblasts obtained from patients affected by Tangier disease have a significant accumulation in cardiolipin content (16-18) led us to hypothesize that ABCA1 deficiency is a major driver of the mitochondrial dysfunction observed in DKD. In fact, a reduction in oxygen consumption rates, increased mitochondrial DNA damage, and reduced mitochondrial oxidative phosphorylation have been described in DKD (19-23). Cardiolipin is a mitochondrial-specific phospholipid that plays an integral role in mitochondrial function and membrane preservation (24). Cardiolipin is also particularly vulnerable to ROS-induced damage, which could in turn contribute to mitochondrial dysfunction $(25,26)$.

In this study, we demonstrate that podocyte-specific deletion of Abca1 in 2 DKD mouse models is sufficient to worsen DKD outcomes. Susceptibility to DKD is not dependent on the accumulation of free cholesterol, as genetic deletion of sterol-o-acyltransferase 1 (SOAT1, which converts free into esterified cholesterol) in podocyte-specific ABCA1-deficient mice is sufficient to cause free cholesterol accumulation but does not cause glomerular injury. ABCA1 deficiency in human podocytes, however, leads to the accumulation of cardiolipin in association with altered function and organization of the mitochondrial respiratory chain (MRC) and inhibiting cardiolipin peroxidation is sufficient to ameliorate DKD in mice. Furthermore, induction of ABCA1, both genetically as shown in vitro and pharmacologically as shown in vivo, is sufficient to ameliorate podocyte injury in DKD. Our findings provide what we believe is the first evidence linking ABCA1 deficiency to cardiolipin accumulation and mitochondrial dysfunction. Our study also provides what we believe is the first evidence that treatment with either a cardiolipin peroxidase inhibitor or a selective ABCA1 inducer may protect from DKD.

\section{Results}

$A B C A 1$ expression is reduced in podocytes exposed to progressor sera in association with increased cytotoxicity. We previously described downregulation of ABCA1 expression in glomeruli from Pima Indians with T2D (9). These data were validated in a second population of patients with T2D, where podocyte lipid droplet accumulation and decreased $\mathrm{ABCA} 1$ expression were described and cor- related with eGFR $(8,9)$. However, the contribution of decreased ABCA1 expression to impaired cholesterol efflux and podocyte injury in DKD remains to be established. Furthermore, it remains to be determined if decreased ABCA1 expression occurs prior to the development of DKD or if it is a consequence of established DKD. To address these 2 questions, we utilized sera collected from Pima Indians to treat cultured human podocytes. Subjects were separated into 2 groups based on measured glomerular filtration rate (mGFR) over 10 years: nonprogressor (NP, patients with the least change in $\mathrm{mGFR}$ ) and progressor ( $\mathrm{P}$, patients with the greatest change in mGFR) (Supplemental Table 1; supplemental material available online with this article; https://oi.org/10.1172/ JCI125316DS1). Sera collected from 2 time points were utilized: baseline, when patients did not have any DKD, and 7 years later, when patients also had a research kidney biopsy. mRNA was isolated and microarray analysis was performed. Gene enrichment analysis identified pathways important in lipid and fatty acid metabolism to be significantly altered between $\mathrm{P}$ and NP patient sera-treated podocytes (Table 1). Key cholesterol metabolism genes were validated by quantitative real-time PCR (qRT-PCR). At time of kidney biopsy, treatment of human podocytes with $\mathrm{P}$ sera caused a significant downregulation of both 3-hydroxy-3-methyl-glutaryl-coenzyme A reductase (HMGCoAR, a protein important in cholesterol synthesis) and ABCA1 when compared with NP treatment. Expression of low-density lipoprotein receptor (LDL-R, a protein important in cholesterol influx) was not affected. However, only a significant downregulation of ABCA1 expression was observed (Figure 1A) in podocytes treated with $P$ sera collected at baseline, suggesting that suppressed ABCA1 expression may occur in podocytes prior to the development of DKD. We next investigated if decreased expression of ABCA1 would lead to impaired apolipoprotein AI-mediated (ApoAI-mediated) cholesterol efflux. We found decreased ApoAI-mediated cholesterol efflux in podocytes treated with $\mathrm{P}$ sera compared with NP sera collected at baseline (Figure 1B). Decreased ApoAI-mediated cholesterol efflux in podocytes treated with $\mathrm{P}$ sera collected at baseline was also associated with increased lipid droplet content as assessed via bodipy (Figure 1, C and D), increased ROS (Figure $1 \mathrm{E}$ ), and cytotoxicity (Figure $1 \mathrm{~F}$ ). Increased cytotoxicity in P seratreated podocytes persisted when sera collected at time of kidney biopsy was utilized (Supplemental Figure 1, A and B). In order to understand if decreased ABCA1 expression, as observed in kidney biopsies and in $P$ sera-treated podocytes, would have any clinical significance, linear regression analyses between ABCA1 expression and serum creatinine, blood urea nitrogen (BUN), mGFR at baseline as well as at 7 years follow-up, albumin-to-creatinine ratio 


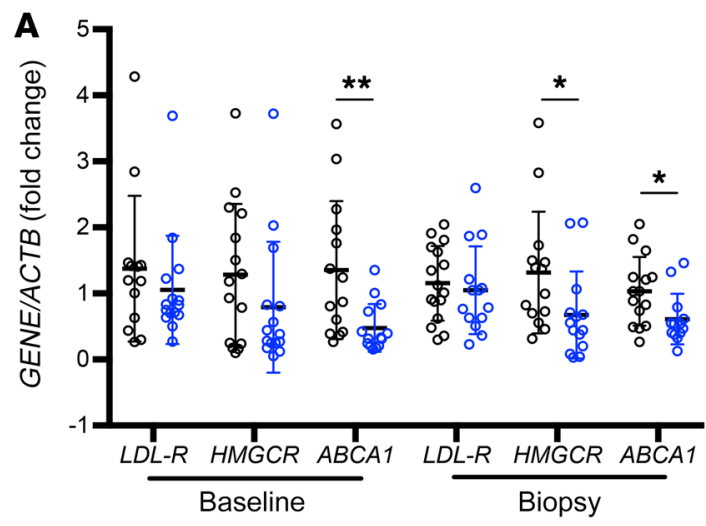

D
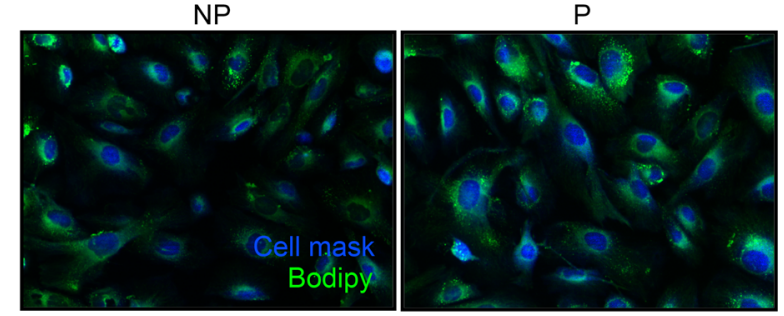

G

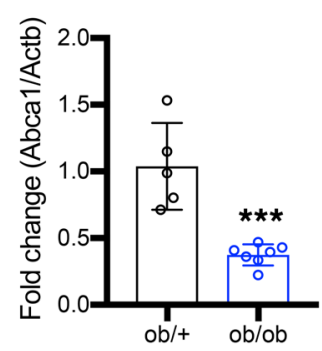

B

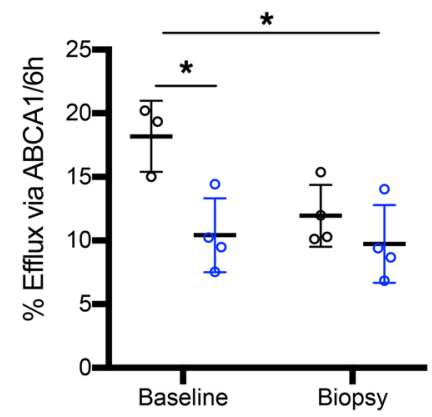

$\mathbf{E}$

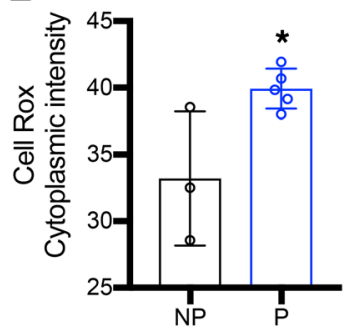

C o Nonprogressors (NP) - Progressors $(P)$

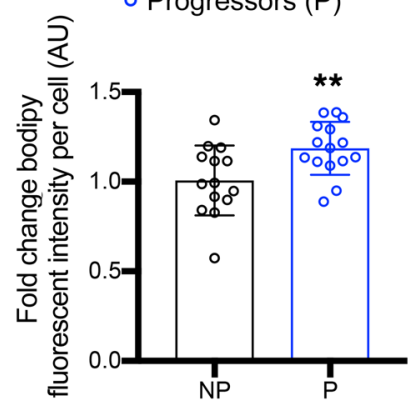

$\mathbf{F}$

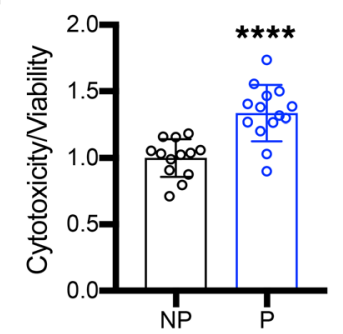

Figure 1. P sera-treated podocytes show reduced ABCA1 expression and function associated with increased cytotoxicity. (A) Expression of several genes important in lipid metabolism was assessed by real-time PCR in NP $(n=13-16)$ and P $(n=14-15)$ sera-treated podocytes, at baseline and at time of biopsy. (B) Quantification of the percentage of radiolabeled cholesterol effluxed via the ABCA1 transporter after treatment with sera from patients obtained at baseline and at time of biopsy. Sera from NP and P patients were used in the analysis ( $n=4$ pooled sera for all groups). (C) Quantification of the lipid droplet content in human podocytes treated with NP and P patient sera via bodipy fluorescent intensity per cell $(n=14-15)$. (D) Representative images (original magnification $\times 20$ ) showing bodipy staining of normal human podocytes treated with the sera from patients with NP and P DKD. (E and F) Bar graph analysis showing CellRox cytoplasmatic intensity ( $n=3$ for NP, $n=5$ for P; pooled sera) (E) and cytotoxicity normalized to viability ( $n=13$ for NP, $n=14$ for P) (F) in human podocytes treated with baseline P sera- compared with NP sera-treated podocytes. (G) Glomerular Abca1 expression of ob/ob mice ( $n=$ 7) compared with their heterozygous controls, ob/+ $(n=4)$ quantified via qRT-PCR. (H) Glomerular Abca1 expression of db/db mice $(n=5)$ compared with their heterozygous controls, $\mathrm{db} /+(n=6)$ quantified via qRT-PCR. (I) Correlation analysis between albumin-to-creatinine ratios ( $\mu \mathrm{g} / \mathrm{mg})$ and glomerular Abca1 expression in $\mathrm{db} / \mathrm{db}$ and ob/ob mice. Two-tailed $t$ test (all panels except I) and linear regression used for correlation analyses (I) with $r^{2}$ and $P$ values shown. ${ }^{*} P<0.05$; ${ }^{* *} P<0.01 ;{ }^{* *} P<0.001 ;{ }^{* * *} P<0.0001$.

(ACR), and glomerular basement membrane thickening (BMT) as an early morphological parameter of DKD were performed (Supplemental Table 2). We found that glomerular ABCA1 expression negatively correlates with patient serum creatinine and BUN levels obtained at time of kidney biopsy, confirming the inverse relationship between glomerular ABCA1 expression and kidney function reported by others (8). Furthermore, ABCA1 expression as observed in podocytes treated with patient sera obtained at baseline correlated negatively with time of biopsy patient ACR and with mean glomerular BMT, indicating that ABCA1 expression levels in podocytes exposed to patient sera could represent a valuable tool to predict DKD, once validated in larger cohorts.
Abca1 expression is reduced in mouse models of DKD. We next examined if decreased Abca1 expression contributes to disease progression using different mouse models of diabetic kidney disease. Abca1 expression was significantly decreased in glomeruli isolated from both BTBR ${ }^{\mathrm{ob} / \mathrm{ob}}$ (Figure $1 G$ ) and B6BKS ${ }^{\mathrm{db} / \mathrm{db}}$ mice (Figure $1 \mathrm{H}$ ) when compared with their heterozygous littermates, ob/+ and $\mathrm{db} /+$, respectively. Correlation analysis showed that glomerular expression of Abca1 negatively correlates with ACR in diabetic mice (Figure 1I), using both the ob/ob and db/db mouse models. Taken together, these data suggest that decreased ABCA1 expression may contribute to DKD progression. However, we previously reported that mice with a podocyte-specific deletion of Abca1 
A

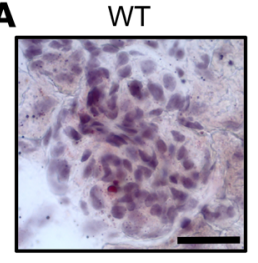

Abca1 ${ }^{f|/ f|}$

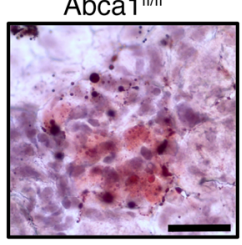

C

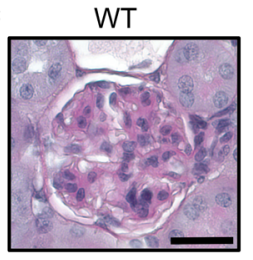

Abca $1^{\mathrm{th/}}$

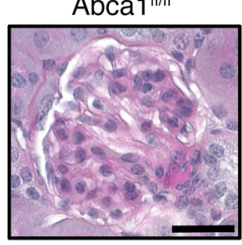

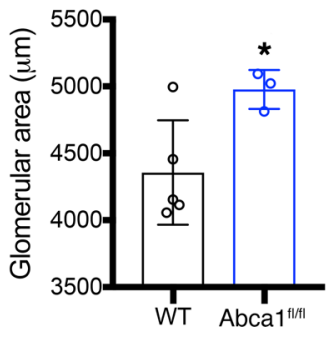

E
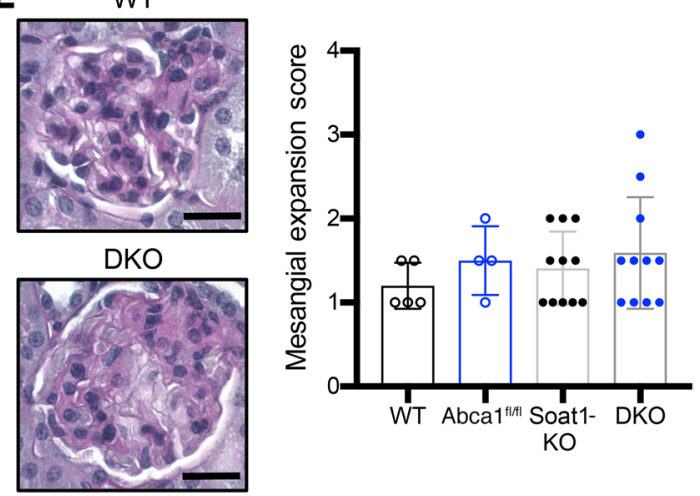

F

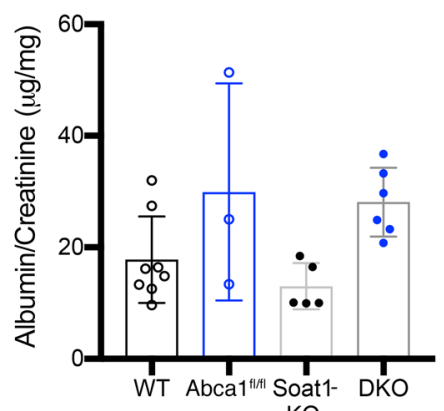

B $\circ W T$

- Abca1 $1^{\text {firt }}$

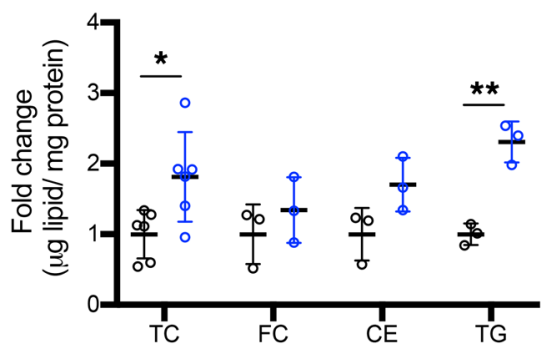

D $\circ W T$

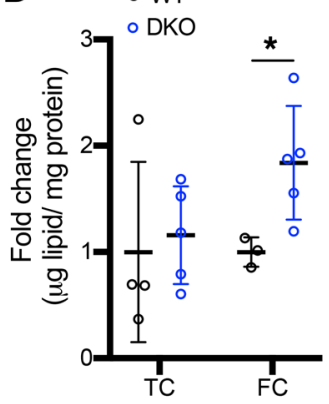

Figure 2. Podocyte-specific Abca1 deficiency leads to lipid accumulation, and concomitant Soat1 deficiency leads to free cholesterol accumulation in the absence of albuminuria. (A) Representative images of oil red 0 -stained kidney cortex sections for the detection of glomerular lipid droplets in WT and Abca $1^{\mathrm{fl} / \mathrm{fl}}$ mice ( $n=3$ per group). (B) Bar graph analysis showing the quantification of total cholesterol (TC), free cholesterol (FC), cholesterol esters (CEs), and triglyceride (TC) levels extracted from kidney cortex of WT $(n=3)$ and podocyte-specific Abca ${ }^{\text {fl/fl }}$ mice $(n=3)$ (fold change in microgram lipid per milligram of protein). (C) Representative images of PAS-stained sections utilized for quantification shown for glomerular area $(\mu \mathrm{m})$ of WT $(n=5)$ and Abca1 $1^{\mathrm{fl} / \mathrm{fl}}(n=3)$ mice. (D) Bar graph analysis showing the quantification of TC and CE content extracted from kidney cortex of WT ( $n=3-4)$ and Abca $1^{f / / f l}$ Soat1 $1^{-/-}(D K O)$ mice $(n=5)$. (E) Representative images for PAS-stained sections of WT $(n=5)$ and DKO $(n=11)$ mice and quantification of the mesangial expansion score. $(F)$ Albu-

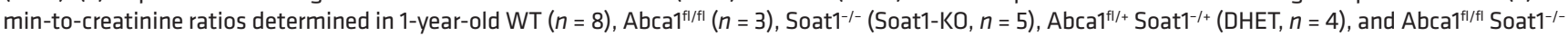
$(\mathrm{DKO}, n=6)$ mice. Scale bars: $25 \mu \mathrm{m}$. Two-tailed $t$ test $(\mathrm{B}-\mathrm{D})$ or 1-way ANOVA followed by Tukey's multiple comparisons test $(\mathrm{E}$ and $\mathrm{F})$. ${ }^{*} P<0.05 ;{ }^{*} P<0.01$.

$\left(\right.$ Abca $\left.^{\mathrm{f} / \mathrm{f}}\right)$ do not develop albuminuria (14), suggesting that additional factors contribute to the pathogenesis of DKD.

Soat1 knockout mice with a podocyte-specific deletion of Abcal have increased free cholesterol and no albuminuria. We previously demonstrated total cholesterol accumulation in experimental DKD $(9,14)$. In light of the fact that podocyte-specific Abca1 knockout mice do not develop albuminuria, we tested the hypothesis that this could be due to accumulation of esterified cholesterol, which is usually considered a neutral and nontoxic form of cholesterol $(27,28)$. We first demonstrated that $\mathrm{Abca1}{ }^{1 / \mathrm{Il}}$ mice are characterized by an increased glomerular lipid droplet content (Figure 2A) when compared with WT mice. Consistent with what was demonstrated in experimental models of DKD $(10,29)$, both total cholesterol and triglycerides were significantly increased in kidney cortexes of $A b c a 1^{\mathrm{A} / \mathrm{l}}$ mice when compared with WT mice. However, although a trend toward increased esterified cholesterol was observed, this trend was not statistically significant (Figure 2B). Nevertheless, the increase in total cholesterol and triglyceride content was associated with a mild increase in the glomerular surface area in $\mathrm{Abca}^{\mathrm{1} / \mathrm{fl}}$ compared with WT mice (Figure 2C). As our prior in vitro findings demonstrated that decreased ABCA1 expression in combination with decreased SOAT1 activity (which prevents esterification of cholesterol) is necessary to induce podocyte injury and loss (14), we tested the hypothesis that accumulation of free cholesterol was needed to cause podocyte injury and proteinuria. $A b c a 1^{\mathrm{A} / \mathrm{l}}$ mice were therefore bred to mice with a constitutive deletion of Soat1 to generate $\mathrm{Abca}^{\mathrm{t} / \mathrm{fl}}$ Soat1 ${ }^{-/-}$(DKO) mice. Interestingly, although these DKO mice did accumulate free cholesterol in the kidney cortex (Figure 2D), they did not develop mesangial expansion or albuminuria at 10 months of age (Figure 2, $\mathrm{E}$ and F) suggesting that free cholesterol kidney cortex accumulation is not sufficient to cause podocyte injury and proteinuria. However, these findings do not exclude the possibility that renal cholesterol accumulation may contribute to DKD development and progression.

Abca1 deficiency is a susceptibility factor in DKD. We next tested the hypothesis that podocyte ABCA1 deficiency is sufficient to confer susceptibility to injury in the context of DKD. First, the previously characterized (14) siRNA ABCA1 knockdown (siABCA1) and siRNA scramble control (siCO) podocytes were treated with NP and P sera obtained from Pima Indians. We observed that siABCA1 podocytes treated with either group of patient sera resulted in significantly increased podocyte cytotoxicity when compared with patient sera-treated siCO (Figure 3A). To establish if ABCA1 deficiency contributes to podocyte injury in DKD, we generated $\mathrm{BTBR}^{\mathrm{ob} / \mathrm{ob}}$ mice with a podocyte-specific deletion of 
A
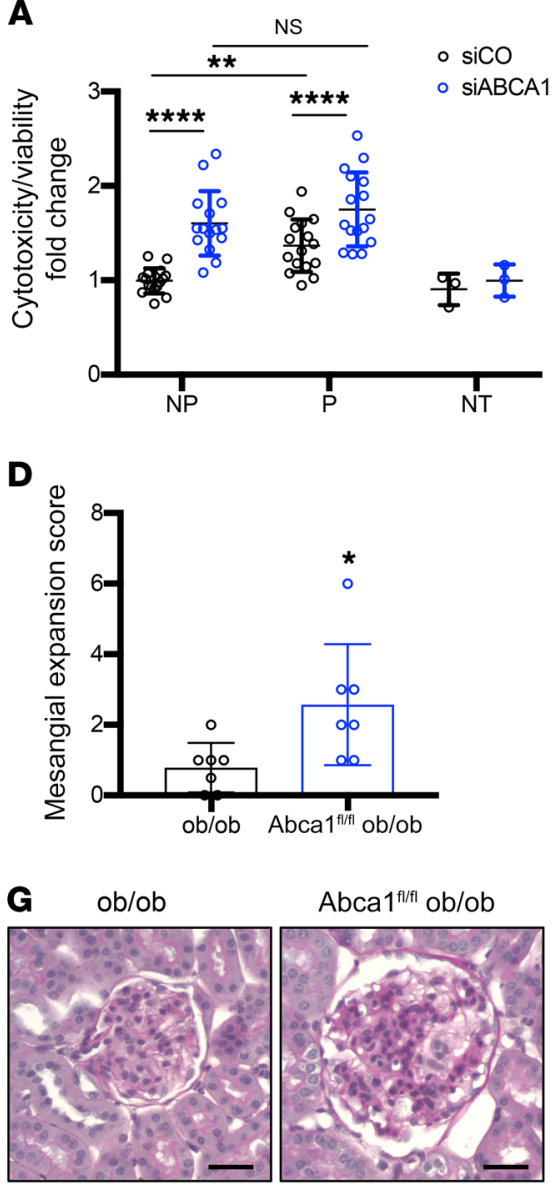

J

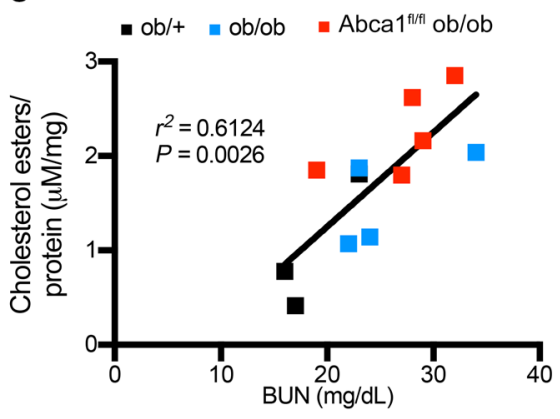

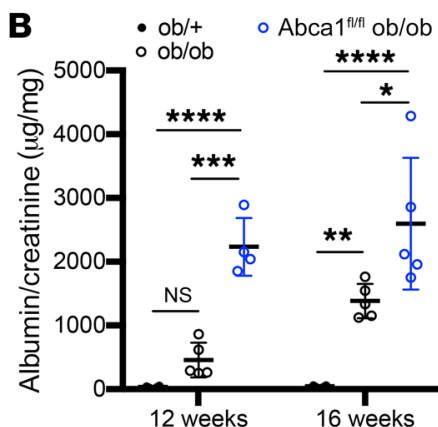

E
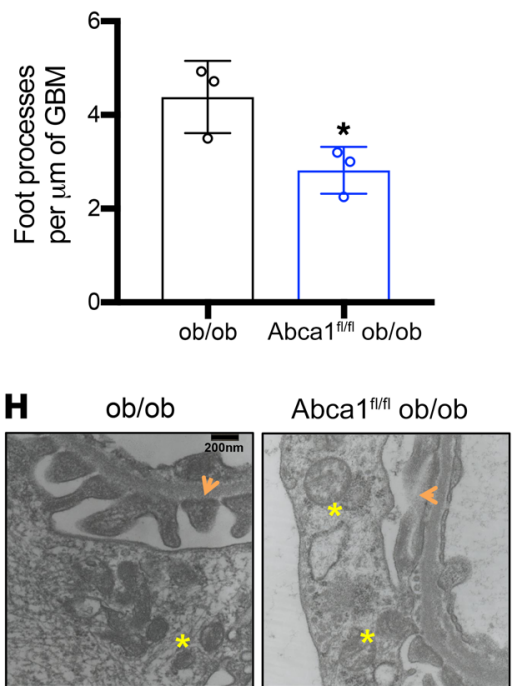

$\mathbf{K}$

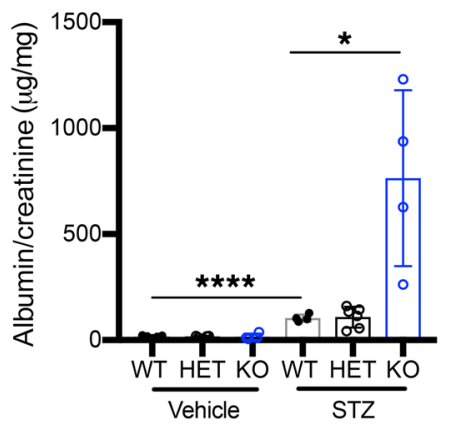

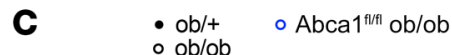
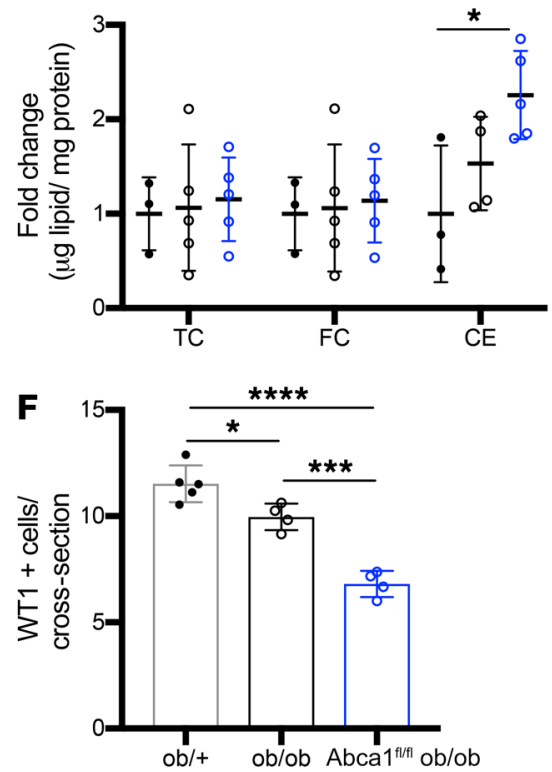

I
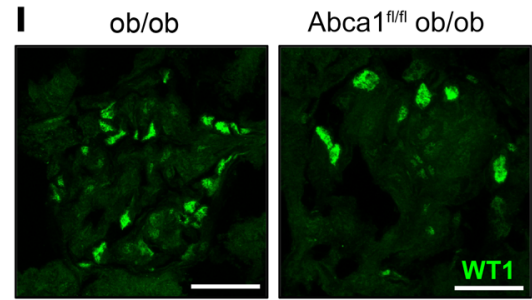

$\mathbf{L}$

$$
\begin{aligned}
& \text { - } K O+V E H \\
& \text { KO + STZ }
\end{aligned}
$$

$\circ W T+S T Z$

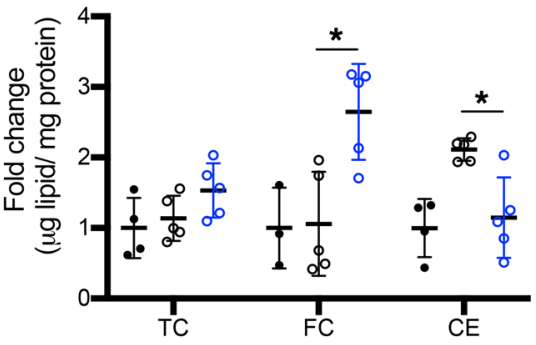

Figure 3. ABCA1 deficiency is a susceptibility factor for podocyte injury contributing to worsened DKD. (A) Quantification for cytotoxicity normalized to viability for NP $(n=16)$ and $\mathrm{P}(n=15)$ sera-treated, or untreated (NT, $n=3)$ siRNA ABCA1 knockdown podocytes (siABCA1) compared with scramble control (siCO) podocytes. (B-F) Ob/+, ob/ob, and $A b c a 1^{\text {fl/ffl }}$ ob/ob mice were analyzed for the following: (B) albumin-to-creatinine ratios at 12 and 16 weeks (time of sacrifice) ( $n=4-5$ per group); (C) TC, FC, and CEs ( $n=3-5$ per group); (D) mesangial expansion score using PAS-stained kidney cortex sections ( $n=7$ per group); (E) podocyte foot processes (marked with orange arrows in $\mathrm{H}$ ) per $\mu \mathrm{m}$ of GBM ( $n=3$ per group); and (F) podocyte number per glomerular cross section as determined via WT1 antibody. (G-I) Representative images for (G) PAS-stained kidney cortex (scale bars: $25 \mu \mathrm{m}$ ); (H) TEM podocyte foot process measurements (mitochondria marked with yellow asterisks; scale bars: $200 \mathrm{~nm}$ ); and (I) WT1-stained kidney cortex sections (scale bars: $25 \mu \mathrm{m}$ ). (J) Correlation analysis between BUN and kidney cortex CEs ( $\mu \mathrm{M}$ cholesterol/mg protein) ( $n=3-5$ per group). (K) Quantification of albumin-to-creatinine ratios comparing WT $(n=4-5)$, Abca ${ }^{\mathrm{fl} /+}(\mathrm{HET})(n=4-6)$, and Abca ${ }^{\mathrm{fl} / \mathrm{fl}}(\mathrm{KO})(n=4)$ mice injected with vehicle or STZ. (L) Quantification of kidney cortex TC, FC, and CEs content in KO and STZ-treated WT and KO mice ( $n=3-5$ per group). Two-tailed $t$ test (A, D, E) or 1-way ANOVA followed by Tukey's multiple comparisons test (B, C, F, K, L) and linear regression used for correlation analyses (J) with $r^{2}$ and $P$ values shown. ${ }^{*} P<0.05 ;{ }^{* *} P<0.01 ;{ }^{* * *} P<0.001$; ${ }^{* * *} P<0.0001$.

Abca1 (Abca1 $\left.1^{\mathrm{f} / \mathrm{fl}} \mathrm{ob} / \mathrm{ob}\right)$. At 12 weeks of age Abca1 $1^{\mathrm{f} / \mathrm{fl}} \mathrm{ob} / \mathrm{ob}$ mice demonstrated increased albuminuria when compared with their diabetic control mice (ob/ob), which persisted until 16 weeks of age (time of sacrifice) (Figure 3B). Abca1 $1^{\mathrm{f} / \mathrm{fl}} \mathrm{ob} / \mathrm{ob}$ mice were found to have an increased cholesterol ester (CE) content in the kidney cortex (Figure 3C) when compared with WT mice. More importantly, we found a strong positive correlation of the CE content in kidney cortex with BUN (Figure 3J). Several serological parameters were analyzed (Supplemental Table 3), and BUN as well as circulating LDL were found significantly elevated in $A b c a 1^{\mathrm{f} / \mathrm{fl}} \mathrm{ob} /$ 
A

$$
\begin{aligned}
& \text { - sico } \\
& \text { - siABCA1 }
\end{aligned}
$$
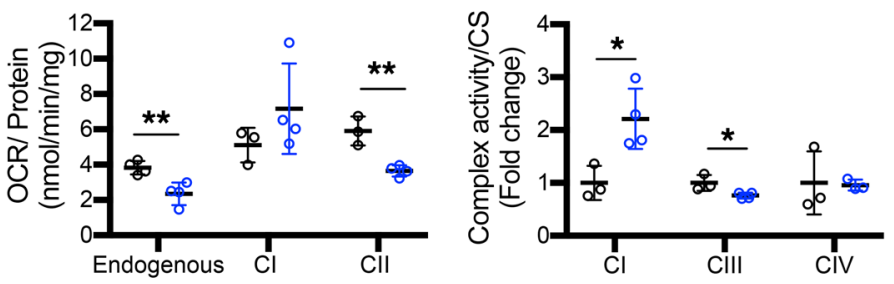

D
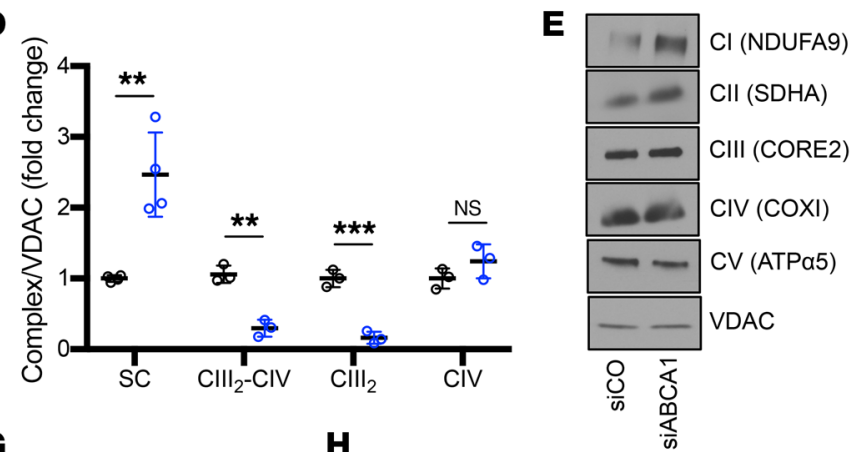

H

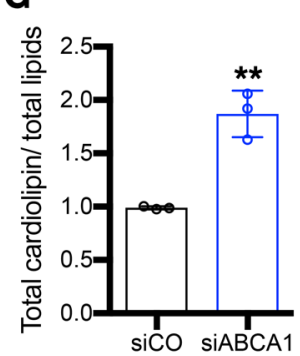

E

B

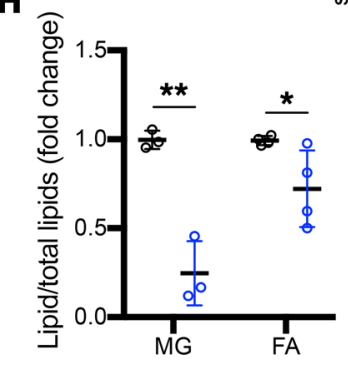

C Exposure time:
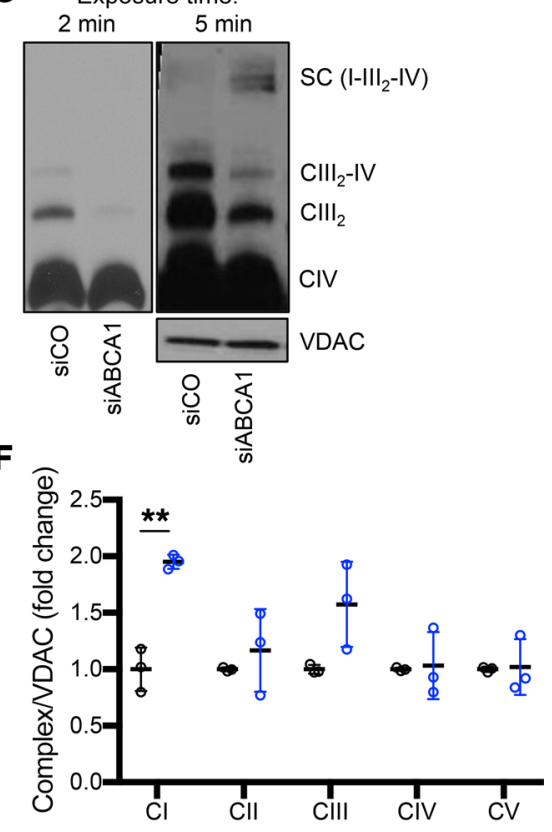

Figure 4. ABCA1 deficiency leads to cardiolipin accumulation associated with reduced mitochondrial function and oxygen consumption. (A and $B$ ) siABCA1 compared with siCO podocytes were analyzed for (A) endogenous (unpermeabilized cells) and substrate-driven (permeabilized cells + glutamatemalate [CI] or succinate [CII]) oxygen consumption rates (OCR; nmol oxygen consumed per minute normalized to mg protein) ( $n=3-4$ per group); (B) maximal enzymatic activity for complex I (CI), complex III (CIII), and complex IV (CIV) ( $n=3-4$ per group). (C) Representative Western blot image of BN-PACE analysis of mitochondrial extracts obtained with digitonin sequentially probed for core 2 antibody (CIII), Cox I antibody (CIV), and SDHA (CII). Samples were also resolved by SDS-PACE and probed with VDAC as loading control. (D) Densiometric quantification of Western blot analysis of digitonin extract shown in C ( $n=3-4$ per group). (E) Representative Western blot images of BN-PAGE analysis of mitochondrial extracts obtained with lauryl maltoside and probed for NDUFA9 (CI), SDHA (CII), CORE2 (CIII), COXI (CIV), and ATP $\alpha 5$ (CV). Samples were also resolved by SDS-PACE and VDAC was detected as loading control. (F) Densiometric quantification of Western blot analysis of lauryl maltoside extraction shown in $\mathrm{E}$ ( $n=3$ per group). (C) Quantification of the relative cardiolipin and (H) monoglycerides (MG) and fatty acids (FA) content normalized to total lipids extracted from isolated mitochondria of siABCA1 compared with siCO podocytes ( $n=3$ per group). Two-tailed $t$ test. ${ }^{*} P<0.05 ;{ }^{* *} P<0.01 ;{ }^{* * *} P<0.001$.

ob mice. PAS staining of kidney cortex sections was performed and revealed an increased mesangial expansion in $\mathrm{Abca} 1^{\mathrm{f} / \mathrm{l}} \mathrm{ob} /$ ob (Figure 3, D and G) compared with ob/ob mice. Using transmission electron microscopy (TEM), we observed Abca1 ${ }^{1 / 1 / 1}$ ob/ob mice to experience a decrease in the number of foot processes per length of the glomerular basement membrane (GBM), as well as mitochondrial swelling when compared with diabetic ob/ob mice (Figure 3, E and H). Mitochondrial swelling is normally observed with alterations in mitochondrial respiration, inner mitochondrial membrane compositions, or fission and fusion (30-32). We therefore investigated whether there were any alterations in fission or fusion and measured glomerular gene expression of fission mitochondrial 1 (Fis1, a protein important in fission), and mitofusin 1 (Mfn1, a protein mediator of mitochondrial fusion).
$A b c a 1^{1 / f / l} \mathrm{ob} / \mathrm{ob}$ mice showed significantly reduced Fis1 and Mfn1 whereas $A b c a 1^{1 / 1 / 1}$ mice only had reduced Fis1 when compared with WT mice (Supplemental Figure 2). These data suggest a role of Abca1 deficiency in contributing to reduced fission and ultimately increased mitochondrial swelling in DKD. Furthermore, WT1 (podocyte-specific antibody) immunofluorescence staining was utilized to quantify podocyte number per glomerular cross section. We found that $\mathrm{Abca} 1^{\mathrm{f} / \mathrm{l}} \mathrm{ob} / \mathrm{ob}$ mice have significantly decreased podocyte numbers per glomerular section when compared with ob/ob mice (Figure 3, F and I). Body and kidney weight (Supplemental Figure 3, A and B) as well as glycemic index (data not shown as all mice were in the undetectable upper range at time of sacrifice, $>600 \mathrm{mg} / \mathrm{dL}$ ) were not altered between ob/ob and $\mathrm{Abca} 1^{\mathrm{t} / \mathrm{f}} \mathrm{ob} / \mathrm{ob}$ mice. To confirm that Abca1 deficiency may 
Table 2. Patient glomerular biopsies, OXPHOS complexes, and FAO gene expression compared with healthy donors

\begin{tabular}{|ccc|}
\hline & Oxidative phosphorylation & \\
\hline OXPHOS complex & Gene subunit & Fold change \\
CI & NDUFA6 & 1.300 \\
& NDUFB3 & 1.145 \\
CII & SDHC & 0.836 \\
\hline CIV & COX6A2 & 0.839 \\
& COX7A1 & 1.340 \\
\hline CV & COX7A2 & 1.135 \\
& ATP5E & 1.169 \\
& ATP5)2 & 1.221 \\
Fene & Fatty acid $\boldsymbol{\beta}$ oxidation \\
& ACADL & Fold change \\
& ACADM & 0.422 \\
ACOX2 & 0.771 \\
\hline
\end{tabular}

All genes were found to be significant with $P<0.05$ after $t$ test with multiple corrections.

confer susceptibility to the development of DKD, WT, Abca1 ${ }^{\mathrm{t} / /+}$ $(\mathrm{HET})$, and $\mathrm{Abca}^{\mathrm{fl} / \mathrm{ll}}(\mathrm{KO})$ mice were injected with streptozotocin (STZ) to induce a type 1-like diabetes. KO mice injected with STZ showed increased albumin-to-creatinine ratios compared with STZ-injected WT mice (Figure 3K), while no differences in glycemia, body weight, and mesangial expansion were observed in any of the STZ-injected groups of mice (Supplemental Figure 3, C-E). Moreover, increased free cholesterol and decreased CEs were observed in kidney cortex of $\mathrm{KO}+\mathrm{STZ}$ mice when compared with the WT+STZ controls (Figure $3 \mathrm{~L}$ ). These data confirm a role for Abca1 deficiency in conferring susceptibility to podocyte injury and worsening DKD outcome in experimental mouse models of DKD. As increased free cholesterol in DKO mice was not sufficient to cause DKD (Figure 2), the exact mechanism by which Abca1 deficiency confers susceptibility to podocyte injury in DKD was further assessed.

ABCA1 deficiency leads to mitochondrial cardiolipin accumulation and mitochondrial dysfunction. Mitochondrial dysfunction is a key feature of $\operatorname{DKD}(22,23,31)$. Many groups have shown that mitochondria in experimental and clinical models of DKD are characterized by altered mitochondrial respiratory chain function, structural and networking anomalies, and increased ROS (19). Since we detected podocyte mitochondrial swelling in $\mathrm{Abca}^{\mathrm{fl} / \mathrm{fl}} \mathrm{ob} / \mathrm{ob}$ mice, we next determined if ABCA1 deficiency in podocytes could contribute to mitochondrial dysfunction. Using siABCA1 podocytes, we found that endogenous (unpermeabilized cells using endogenous substrates) and complex II (CII) substrate-driven (permeabilized cells with exogenous addition of succinate) oxygen consumption was significantly reduced in siABCA1 podocytes when compared with siCO (Figure 4A). However, no differences in ATP content were observed (Supplemental Figure 4A). The maximal enzymatic activity of individual complexes was assessed spectrophotometrically, and we observed complex I (CI) activity to be significantly increased, whereas complex III activity was significantly reduced and complex IV (CIV) activity remained unchanged in siABCA1 compared with siCO podocytes (Figure 4B, Supplemental Figure $4 \mathrm{~B}$ ). In order to understand if the observed decrease in oxygen consumption rates and OXPHOS activities was due to alterations in mitochondrial OXPHOS complexes, mitochondrial enriched lysates were analyzed by Blue Native-PAGE and Western blot. We observed a remodeling of the OXPHOS complexes. Specifically, mitochondrial membrane extracts obtained in the presence of the detergent digitonin, known to preserve OXPHOS complex interactions, presented with an increase in supercomplex, or respirasome $\mathrm{I}-\mathrm{III}_{2}-\mathrm{IV}$, in combination with decreases in complex $\mathrm{III}_{2}$-IV and complex $\mathrm{III}_{2}$, and no differences in complex IV (Figure 4, C and D) were observed. Complex IV was quantified based on a lower exposure time (Figure 4D, Supplemental Figure 4C). Moreover, we did not observe any alterations in the steady state levels of individual OXPHOS complexes with the exception of an increase in complex I in siABCA1 compared with siCO podocytes (Figure 4, E and F). Taken together, these data suggest that ABCA1 deficiency contributes to podocyte susceptibility via the remodeling of OXPHOS complexes and the alteration of mitochondrial lipid composition, without changes in ATP production. No differences were detected in mitochondrial membrane potential between siABCA1 and siCO podocytes but some subtle changes in the mitochondrial network distribution were apparent (Supplemental Figure 4, D and E). Furthermore, siABCA1 podocytes experienced a significant increase in superoxide dismutase 2 (SOD2, a mitochondrial specific ROS scavenger) expression (Supplemental Figure 5, A and B), suggesting an increase in mitochondrial-produced ROS. Lipid content of isolated mitochondria from siABCA1 podocytes compared with siCO podocytes were assessed via mass spectrometry and various lipid species were significantly modulated. Most interestingly, total cardiolipin content was significantly increased and monoglycerides and fatty acids were significantly reduced in isolated mitochondria from siABCA1 podocytes (Figure 4, G and $\mathrm{H}$, Supplemental Table 4). Reduction of endogenous cell respiration and complex III activity despite the increase in CI and CI-containing supercomplex levels together with alterations in mitochondrial fatty acid content prompted us to investigate the status of fatty acid beta oxidation (FAO), which was previously described to be impaired in kidney disease (33). qRT-PCR was carried out for several key genes involved in beta oxidation in both patient seratreated podocytes and Abca1 ${ }^{\mathrm{f} / \mathrm{fl}}$ mice. Peroxisome proliferator-activated nuclear receptor alpha (PPARA), acyl-coA dehydrogenase medium chain (ACADM), and acyl-coA oxidase 1 or 2 (ACOX1/2) were significantly downregulated in both $\mathrm{P}$ sera-treated podocytes when compared with NP and in Abca $1^{\mathrm{f} / \mathrm{fl}}$ mice when compared with WT mice (Supplemental Figure 6, A and B). ACADM was also found to be significantly reduced in siABCA1 podocytes (Supplemental Figure 6C). To confirm that these observations are of clinical relevance, we assessed the expression profiles of genes important in OXPHOS and FAO in glomerular biopsies from Pima Indian patients with DKD, in which we previously described significant downregulation of ABCA1 expression (9). We found a significant downregulation in the expression of genes important in FAO and of genes encoding OXPHOS subunits of CII, while genes encoding subunits of CI, CIV, and complex V (CV) were upregulated, supporting our findings in siABCA1 podocytes (Table 2). 

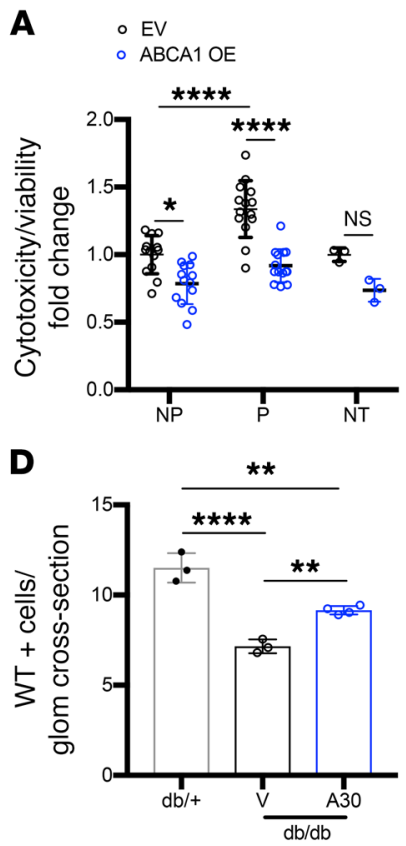

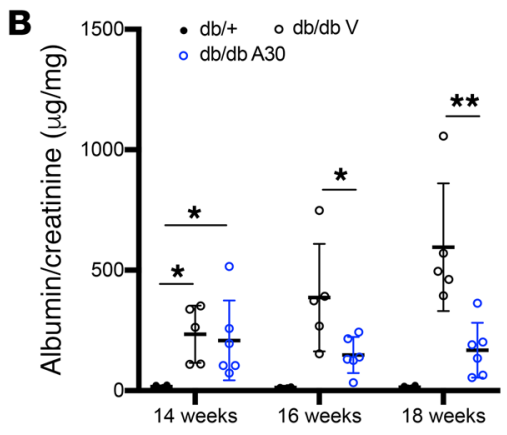

E

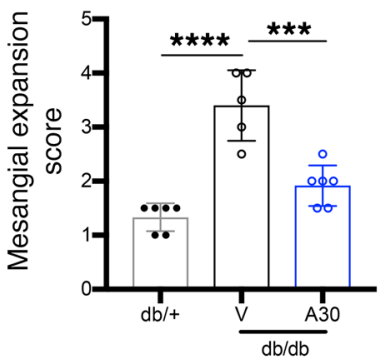

$\mathrm{db} / \mathrm{db}$
G
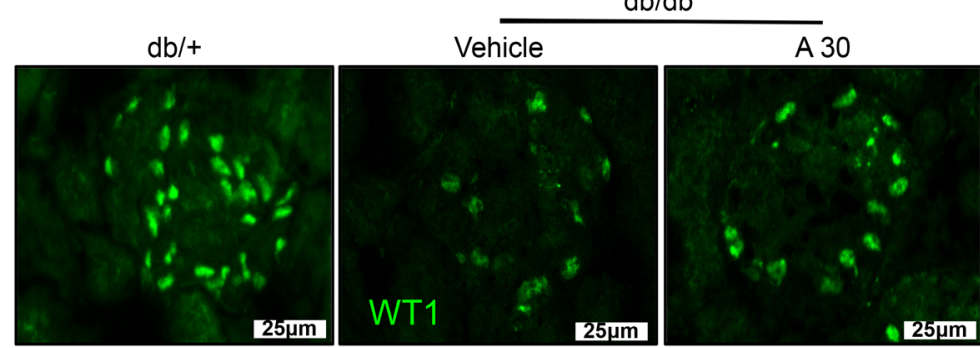

$\mathbf{H}$

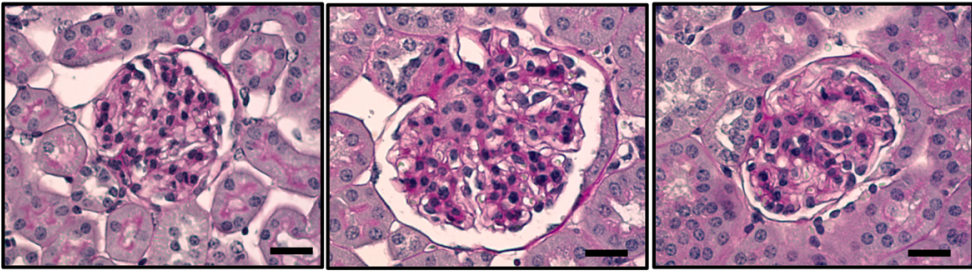

I

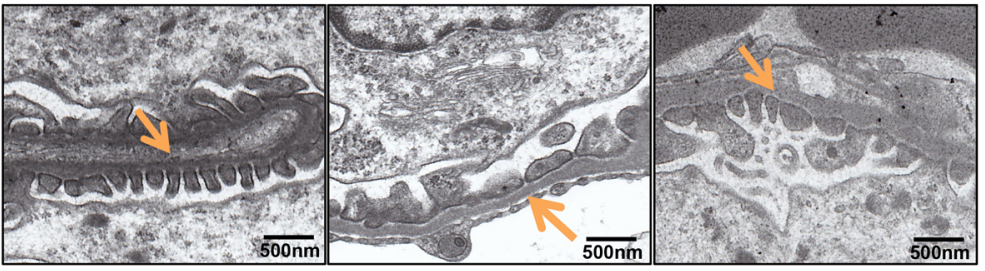

C
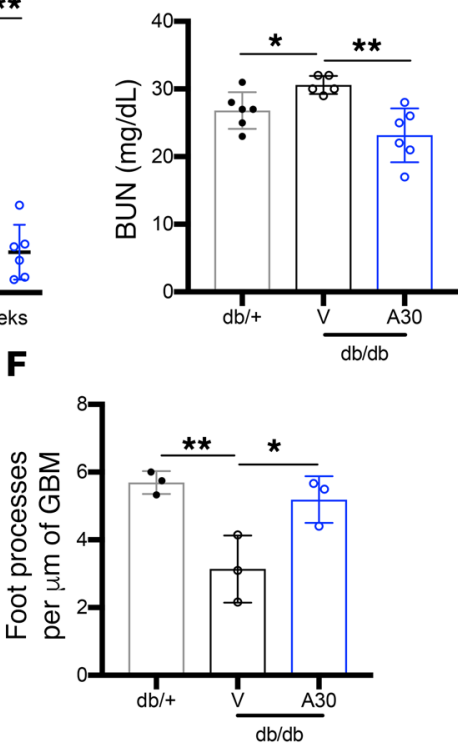
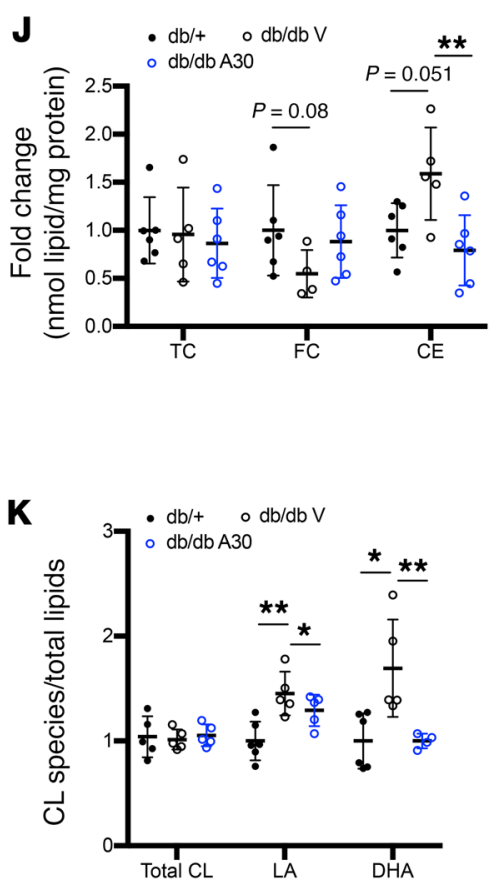

Figure 5. Treatment with an Abca1 inducer (A30) ameliorates podocyte injury and DKD. (A) Quantification of cytotoxicity normalized to viability for NP $(n=13)$ and $\mathrm{P}(n=14)$ sera-treated, or untreated (NT, $n=3)$ ABCA1-overexpressing (ABCA1 OE) podocytes compared with empty vector transfected (EV) podocytes. (B-F) Db/+, vehicle-treated db/db, and Abca1 inducer-treated (A30-treated) db/db mice were analyzed for (B) albumin-to-creatinine ratios determined at start of treatment (14 weeks), after 2 weeks of treatment (16 weeks), and at time of sacrifice after 4 weeks of treatment (18 weeks) ( $n=5-6$ per group); (C) BUN ( $n=5-6$ per group); (D) podocyte number per glomerular cross section ( $n=3-4$ per group) determined by WT1 staining; (E) mesangial expansion score quantification determined from PAS staining ( $n=5-6$ per group); and (F) podocyte foot processes per $\mu \mathrm{m}$ of CBM ( $n=3$ per group) determined from TEM images. (G-I) Representative images of (G) WT1 staining (scale bars: $25 \mu \mathrm{m}$ ); (H) PAS staining (scale bars: $25 \mu \mathrm{m}$ ); and (I) TEM to identify podocyte foot processes (marked with orange arrows) per $\mu \mathrm{m}$ of CBM (scale bars: $500 \mathrm{~nm}$ ). (J) Kidney cortex cholesterol content determination in form of TC, FC, and CEs ( $n=5-6$ per group). (K) Quantification of the relative cardiolipin species rich in linoleic acid (LA) or docosahexaenoic acid (DHA) analyzed via mass spectrometry and normalized to total lipids extracted from $\mathrm{db} /+, \mathrm{db} / \mathrm{db}$ vehicle, and $\mathrm{db} / \mathrm{db}$ A30 kidney cortex ( $n=5-6$ per group). Two-tailed $t$ test (A) or 1-way ANOVA followed by Tukey's test (all other panels). ${ }^{*} P<0.05 ;{ }^{* *} P<0.01 ;{ }^{* * *} P<0.001 ;{ }^{* * *} P<0.0001$. 

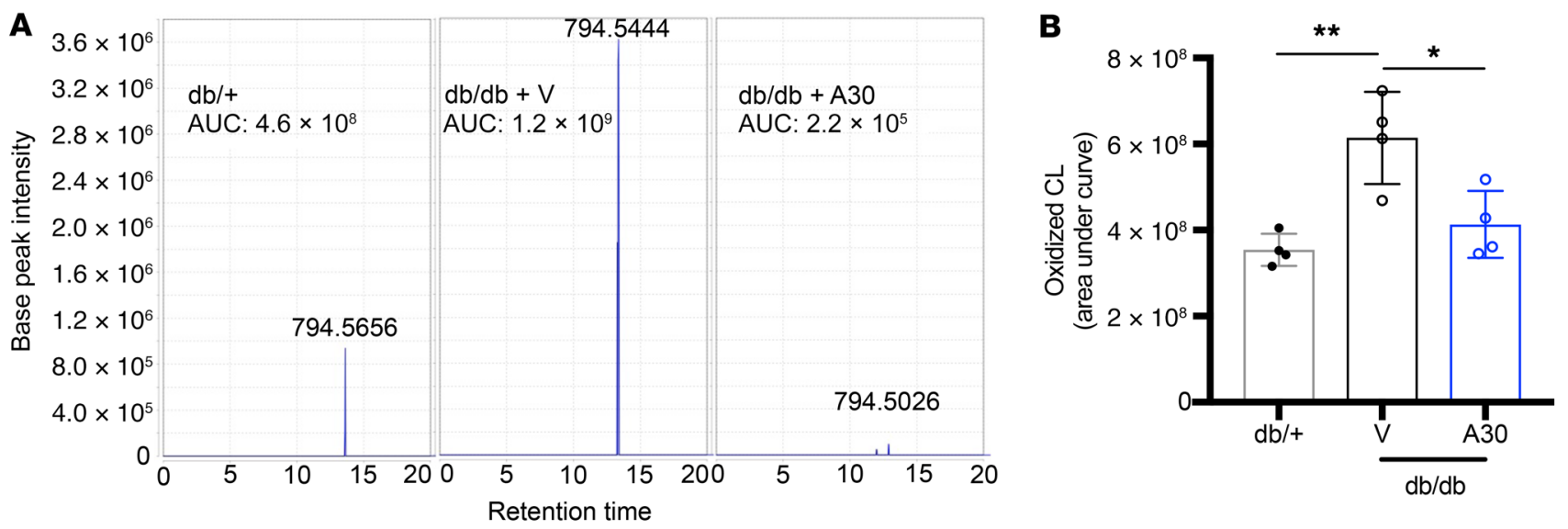

Figure 6. A30 treatment results in reduced cardiolipin oxidation. (A) Representative images of peak intensity for the specific cardiolipin species found at the $\mathrm{m} / \mathrm{z} 794.5$ (number showcased above the peak), comparing db/+ (first panel), $d b / d b$ vehicle-treated mice (second panel), and db/db A30-treated mice (third panel). AUC for each representative peak is annotated. (B) Bar graph analysis of total oxidized cardiolipin species comparing db/+ controls, db/db vehicle-treated, and A30-treated mice ( $n=4$ per group). One-way ANOVA followed by Tukey's test. ${ }^{*} P<0.05 ;{ }^{* *} P<0.01$.

Induction of $A B C A 1$ reverts podocyte injury and $D K D$. Our findings suggest that $\mathrm{ABCA} 1$ deficiency is a key factor driving podocyte injury in DKD. To confirm this observation, we determined whether increasing ABCA1 expression and/or activity in DKD could represent a treatment option in vitro and in vivo. Using our previously characterized $A B C A 1$ overexpressing human podocytes (14), we investigated if ABCA1 overexpression could protect from $\mathrm{P}$ sera-mediated cytotoxicity. ABCA1 overexpressed (OE) podocytes experience decreased cytotoxicity in both NP and P seratreated podocytes when compared with the empty vector control (EV) (Figure 5A). Furthermore, ABCA1 OE podocytes experienced a significant reduction in lipid droplet content when treated with patient sera from NP and P (Supplemental Figure 7A). Because we observed a protection from P-mediated cytotoxicity in ABCA1 OE podocytes, we next determined expression levels of the fatty-acid beta oxidation genes and found PPARA, ACADM, and ACOX2 to be significantly increased in $\mathrm{P}$ sera-treated $\mathrm{ABCA1} O \mathrm{OE}$ podocytes (Supplemental Figure 7B). To validate ABCA1 as a therapeutic target for DKD in vivo, $\mathrm{db} / \mathrm{db}$ mice were treated with a pharmacological inducer of ABCA1 (A30)(34). Db/db mice were treated with $\mathrm{A} 30$ at 14 weeks of age and experienced a significant reduction in albuminuria after 2 weeks of treatment (16 weeks) and an even further reduction after 4 weeks of treatment (18 weeks) (Figure 5B). No changes were observed in glycemia or in body weight between vehicle and A30-treated $\mathrm{db} / \mathrm{db}$ mice (Supplemental Figure 8, A and B). A30 treatment also improved BUN levels in A30-treated db/db mice (Figure 5C). Immunofluorescence detection of WT1 showed a reduction in podocyte number of $\mathrm{db} / \mathrm{db}$ mice whereas the podocyte number in mice treated with A30 remained comparable but still less than $\mathrm{db} /+$ mice (Figure 5, D and G). Mesangial expansion was significantly reduced with $\mathrm{A} 30$ treatment as shown in the PAS-stained kidney cortex sections (Figure 5, E and H). A30 treatment also improved the number of foot processes per length of GBM in db/ $\mathrm{db}$ mice (Figure 5, F and I). To confirm that improved renal function in $\mathrm{db} / \mathrm{db}$ A30-treated mice was associated with increased ABCA1 expression, $\mathrm{ABCA} 1$ protein expression levels were determined in lysates of kidney cortexes of $\mathrm{db} /+, \mathrm{db} / \mathrm{db}$ vehicle-treated, and $\mathrm{db} /$ $\mathrm{db}$ A30-treated mice. Vehicle-treated $\mathrm{db} / \mathrm{db}$ mice showed reduced ABCA1 expression compared with $\mathrm{db} /+$ mice, which was restored in A30-treated db/db mice (Supplemental Figure 9, A and B). Cholesterol quantification of kidney cortexes from $\mathrm{db} / \mathrm{db}$ mice treated with $\mathrm{A} 30$ experience a significant reduction in the $\mathrm{CE}$ content when compared with vehicle-treated $\mathrm{db} / \mathrm{db}$ controls (Figure 5J). Correlation analysis was performed and demonstrated a significant positive correlation between kidney cortex CE content and BUN in db/+, $\mathrm{db} / \mathrm{db}$ A30, and db/db vehicle mice (Supplemental Figure 9C). The beneficial effect of A30 treatment in DKD occurred in association with a reduction of selective cardiolipin species. While both linoleic acid-rich (LA-rich) and docosahexaenoic acid-rich (DHA-rich) cardiolipin species were increased in kidney cortex from $\mathrm{db} / \mathrm{db}$ mice compared with the $\mathrm{db} /+$ controls, treatment with $\mathrm{A} 30$ only reduced the accumulation of DHA without changes to the total cardiolipin content (Figure 5K). Next, we assessed the amount of peroxidized cardiolipin species in the kidney cortexes of A30-treated $\mathrm{db} / \mathrm{db}$ mice compared with $\mathrm{db} / \mathrm{db}$ vehicle-treated mice and $\mathrm{db} /+$ controls via lipid database analysis. We found that peroxidized cardiolipin species were increased in $\mathrm{db} / \mathrm{db}$ vehicle-treated mice while A30 treatment prevented this increase (Figure 6, A and B, Supplemental Figure 10). These data suggest that renoprotection of $A B C A 1$ inducers may be linked to modulation of DHA-rich cardiolipin species that have been previously reported to enhance oxidative stress and to be upregulated in diabetes (35-37), which may further reduce the oxidation of cardiolipin.

Elamipretide treatment ameliorates podocyte loss and DKD. We next aimed at understanding the contribution of cardiolipin to DKD. We first tested if treatment with elamipretide, which stabilizes cardiolipin at the inner mitochondrial membrane and inhibits cytochrome c peroxidase mediated cardiolipin peroxidation (38), could revert DKD progression in $\mathrm{BTBR}^{\mathrm{ob} / \mathrm{ob}}$ mice. In order to design a clinically relevant treatment strategy, $\mathrm{BTBR}^{\mathrm{ob} / \mathrm{ob}}$ mice were aged to 16 weeks, a time point in which these mice have advanced albuminuria, hyperglycemia, and show podocyte loss (39). At this time, 
A

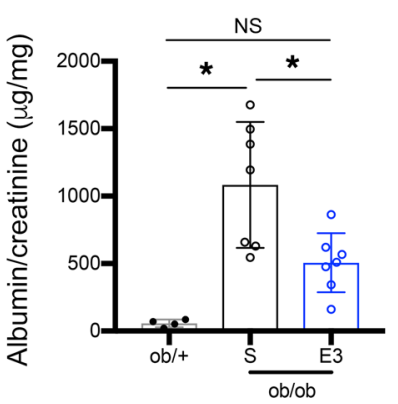

D

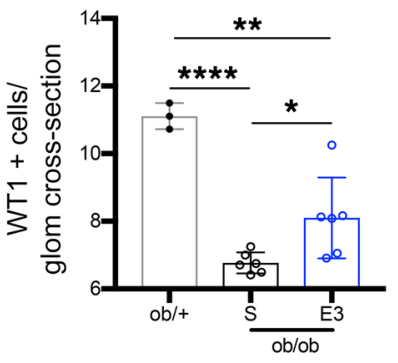

B

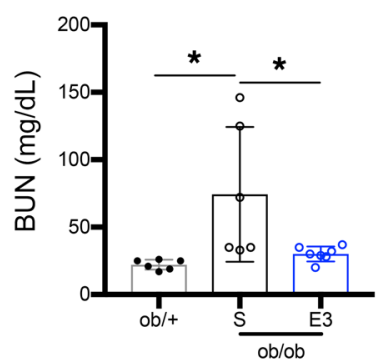

E

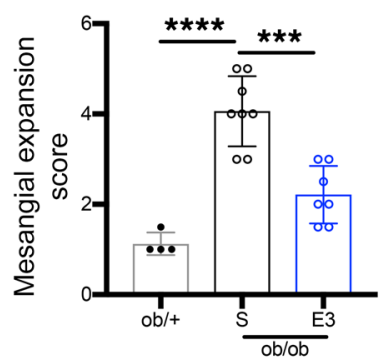

C

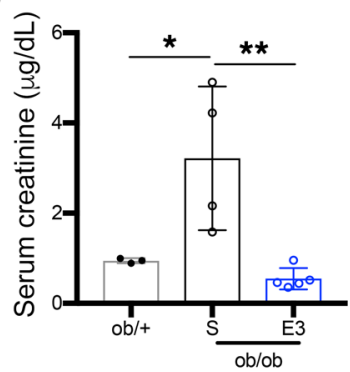

$\mathbf{F}$

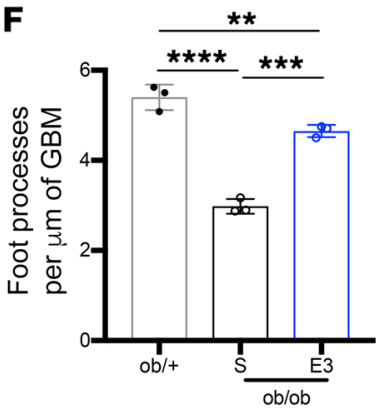

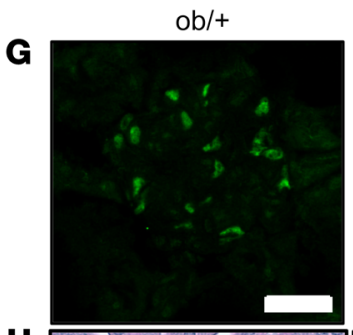
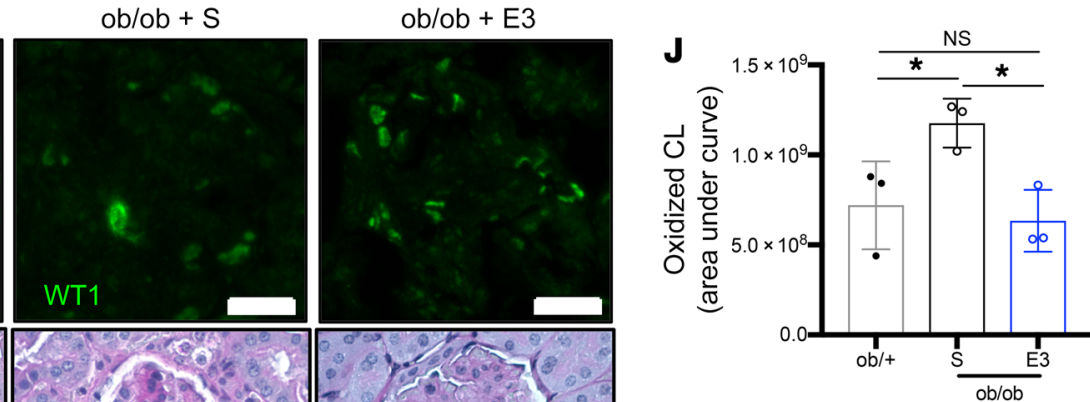

H
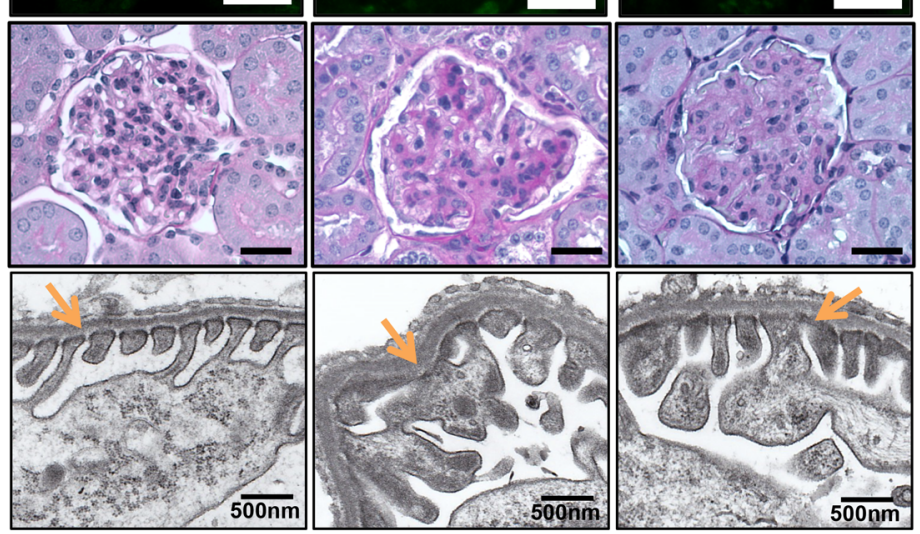

$\mathbf{K}$

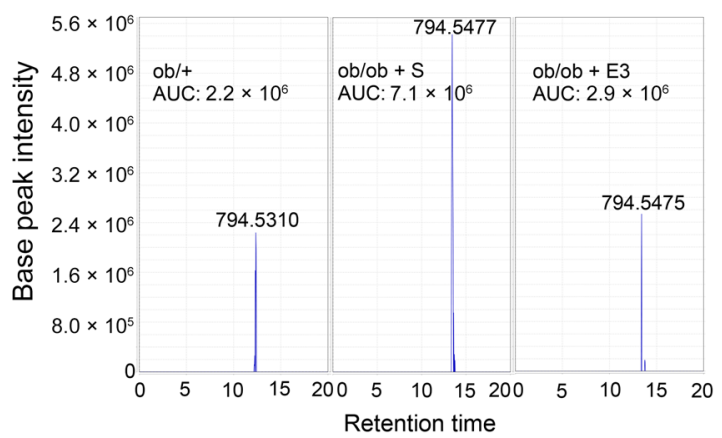

Figure 7. Inhibition of cardiolipin peroxidation ameliorates podocyte injury and DKD in ABCA1 deficient models. (A-F) Saline treated (S) Ob/+ and ob/ob mice, and E3-treated ob/ob mice were analyzed for (A) albumin-to-creatinine ratios determined after 4 weeks of treatment (20 weeks, time of sacrifice) ( $n=4-7$ per group); (B) BUN ( $n=6-7$ per group); (C) serum creatinine ( $n=3-5$ per group); (D) podocyte number per glomerular cross section ( $n=3-6$ per group) determined by WT1 staining; (E) mesangial expansion score quantification determined from PAS staining ( $n=4-8$ per group); and (F) podocyte foot processes per $\mu \mathrm{m}$ of CBM ( $n=3$ per group) determined from TEM images. (G-I) Representative images of (G) WT1 staining (scale bars: $25 \mu \mathrm{m})$; (H) PAS staining (scale bars: $25 \mu \mathrm{m}$ ); and (I) TEM to identify podocyte foot processes (marked with orange arrows) per $\mu \mathrm{m}$ of GBM (scale bars: $500 \mathrm{~nm}$ ). (J) Bar graph analysis of total oxidized cardiolipin species comparing ob/+ controls, ob/ob saline-treated, and E3-treated mice ( $n=3$ per group). (K) Representative images of peak intensity for the specific cardiolipin species found at the $\mathrm{m} / \mathrm{z} 794.5$ (number showcased above the peak), comparing ob/+ (first panel), ob/ ob saline-treated mice (second panel), and ob/ob E3-treated mice (third panel). AUC for each representative peak is annotated. One-way ANOVA followed by Tukey's test. ${ }^{*} P<0.05$; ${ }^{* *} P<0.01$; ${ }^{* *} P<0.001 ;{ }^{* * *} P<0.0001$.

mice were treated daily with elamipretide (E3) for 4 weeks and sacrificed at 20 weeks of age. BTBR ${ }^{\mathrm{ob} / \mathrm{ob}}$ mice treated with $\mathrm{E} 3$ for 4 weeks showed a significant reduction in ACR (Figure 7A), BUN (Figure $7 \mathrm{~B}$ ), and serum creatinine (Figure $7 \mathrm{C}$ ) when compared with $\mathrm{BTBR}^{\mathrm{ob} / \mathrm{ob}}$ saline-treated mice. Histological assessment of
E3-treated BTBR ${ }^{\mathrm{ob} / \mathrm{bb}}$ kidney sections demonstrated a significant increase in podocyte number as detected by WT1 (Figure 7, D and $\mathrm{G}$ ), a significant reduction in mesangial expansion as detected by PAS staining (Figure 7, E and H), and restored podocyte foot processes as shown by TEM (Figure 7, $\mathrm{F}$ and I) when compared with 
A
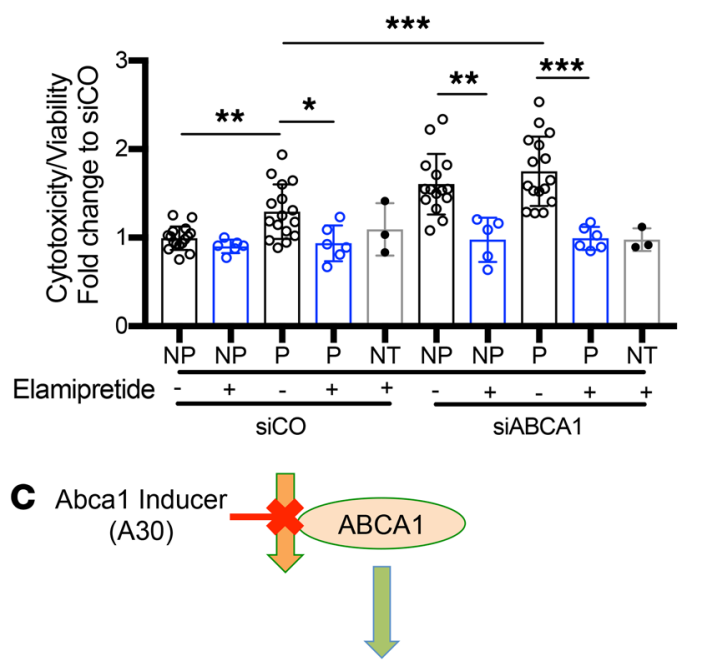

Lipid accumulation

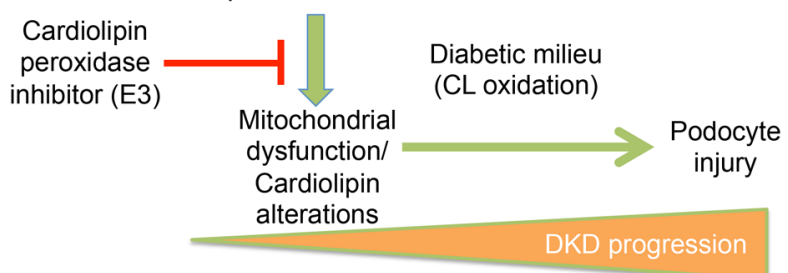

B

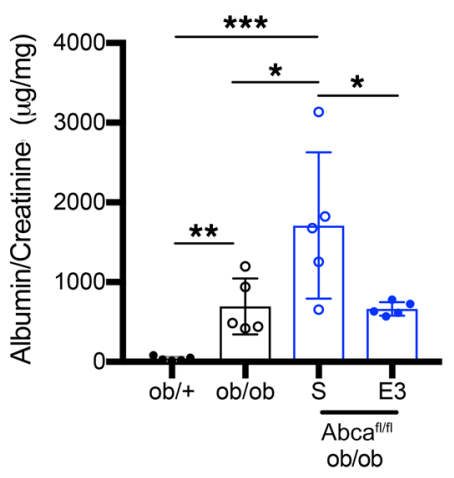

Figure 8. Inhibition of cardiolipin peroxidation reverts $A B C A 1$ deficiency-mediated podocyte injury. (A) Quantification of cytotoxicity normalized to viability in SiABCA1 and siCO podocytes treated with NP and $P$ sera and cotreated with $\mathrm{E3}$, or not treated with $\mathrm{E} 3$ ( $\mathrm{n}=5-16$ pooled sera per group). (B) Albumin-to-creatinine ratios determined after 4 weeks of treatment in 16-week-old ob/+, ob/ob, Abca $1^{\mathrm{fl} / \mathrm{fl}}$ ob/ob saline-treated (S) and Abca1 ${ }^{f / / f 1}$ ob/ob E3-treated mice ( $n=5$ per group). (C) Schematic representation of the current working model and major conclusions drawn from this study. One-way ANOVA followed by Tukey's test. ${ }^{*} P<0.05$; ${ }^{* *} P<0.01 ;{ }^{* *} P<0.001$. saline-treated $\mathrm{BTBR}^{\mathrm{ob} / \mathrm{ob}}$ mice. To confirm that the improvement in DKD progression was associated with inhibition of cardiolipin oxidation, we analyzed oxidized cardiolipin species of total lipids extracted from the kidney cortex of E3-treated ob/ob mice as compared with saline-treated mice. Increased oxidation of cardiolipin was observed in ob/ob mice compared to ob/+ mice; however, E3 treatment prevented the oxidation of cardiolipin in ob/ob mice (Figure 7, J and K, Supplemental Figure 12). We then investigated if ABCA1 dependent susceptibility to DKD is mediated by cardiolipin. In order to do so, P sera-treated siABCA1 podocytes and Abca $1^{\mathrm{t} / \mathrm{l}}$ $\mathrm{ob} / \mathrm{ob}$ mice were treated with E3. NP and P sera-mediated podocyte cytotoxicity was prevented in E3-treated siABCA1 podocytes (Figure 8A). In addition, treatment of 12 -week-old Abca1 ${ }^{\mathrm{f} / \mathrm{fl}} \mathrm{ob} / \mathrm{ob}$ mice with $\mathrm{E} 3$ for 4 weeks was sufficient to rescue them from their increased susceptibility to albuminuria (Figure $8 \mathrm{~B}$ ). Treatment of Abca1 ${ }^{\mathrm{fl} / \mathrm{fl}}$ ob/ob mice with E3 also improved mesangial expansion, podocyte numbers (Supplemental Figure 11, A-C), and overall mitochondrial morphology and structure compared with saline control mice (Supplemental Figure 11D). These data collectively suggest that ABCA1 deficiency contributes to mitochondrial dysfunction by impairing proper cardiolipin content and function, thus rendering podocytes susceptible to injury in a diabetic milieu. Upregulation of ABCA1 or inhibition of cardiolipin peroxidation are both sufficient to improve DKD progression and revert podocyte injury, as shown in our current working model (Figure $8 \mathrm{C}$ ).

\section{Discussion}

This study demonstrates for the first time how ABCA1 deficiency may link lipid metabolism to mitochondrial dysfunction and contribute to podocyte injury and DKD progression. We identified a new mechanism by which ABCA1 deficiency leads to cardiolipin accumulation and alterations in mitochondrial oxidative phosphorylation, resulting in podocyte injury and worsened DKD.

While a reduction of glomerular ABCA1 expression was already reported in 2 separate cohorts of patients with T2D and was found to be correlated with eGFR $(8,9)$, the cell-based assay utilized in this study allowed us to demonstrate that suppression of ABCA1 expression in podocytes occurs prior to the onset of DKD in patients susceptible to DKD progression. Correlation analyses of gene expression from glomerular biopsies or of podocytes treated with the sera from patients with clinical parameters was performed, and demonstrated that ABCA1 expression inversely correlated with BUN, serum creatinine, and ACR (Supplemental Table 2). Podocytes exposed to patient sera obtained at baseline (prior to the onset of DKD) negatively correlated with patient ACR and glomerular BMT at time of biopsy, suggesting ABCA1 expression to be an early phenomenon in the pathogenesis of DKD that could be used to predict DKD progression, once validated in larger cohorts with research kidney biopsies and longitudinal clinical data. In addition, while only the treatment of podocytes with sera from patients with progressive but not with nonprogressive DKD causes ROS accumulation and cytotoxicity, reduction of ABCA1 expression via siRNA was sufficient to cause cytotoxicity, even when nonprogressive sera were used. In line with these observations, genetic ABCA1 overexpression in vitro prevented $\mathrm{P}$ seramediated podocyte injury, while in vivo pharmacologic induction of Abca1 improved existing DKD. We confirmed these observations using ob/ob mice with a podocyte-specific deletion of Abca1 as a novel mouse model of T2D as well as a STZ-driven mouse model of T1D. Abca1 deficiency resulted in a worsened histolog- 
ical, morphological, and serological phenotype in the T2D model and only worsened albuminuria in the T1D model. Taken together, these data suggest that ABCA1 deficiency may represent a molecular switch from nonprogressive to progressive DKD. Decreased ABCA1 expression in DKD is not a specific finding for podocytes, as similar findings were also described in mesangial and tubular cells in a STZ model of T1D (40).

In order to assess how ABCA1 deficiency confers susceptibility to worsened DKD, we investigated the lipid content, oxygen consumption rate, and OXPHOS levels and function in vitro in siABCA1 podocytes. We demonstrated increased cellular lipid content, reduced respiratory capacity associated with alterations in mitochondrial OXPHOS complexes, and increased cardiolipin content in vitro in siABCA1 podocytes compared with siCO. These findings were further validated in the glomerular biopsies of DKD patients from the Pima Indian cohort. More importantly, we demonstrated that targeting cardiolipin, in both in vitro and in vivo models, was beneficial in the treatment of DKD. ROS-induced cardiolipin peroxidation is detrimental to mitochondrial function $(24,41,42)$. While cardiolipin accumulation was observed in podocytes with ABCA1 deficiency, this accumulation is not detrimental to the cell as no decrease in podocyte viability was observed. Instead, our data demonstrate that cardiolipin accumulation is associated with and may contribute to the alterations observed in the mitochondria OXPHOS complexes, thus rendering podocytes susceptible to oxidative injury in DKD. Previous studies have shown that modifications of the electron transport chain which render the system less efficient contributes to increased ROS production (43-45). Indeed, both clinical and experimental DKD are characterized by elevated ROS (45-49). For example, glomeruli isolated from $\mathrm{db} / \mathrm{db}$ mice have increased ROS production when compared with $\mathrm{db} /+$ mice (50). Therefore, it seems possible that in a diabetic environment where ROS is elevated, ABCA1 deficiency-mediated cardiolipin accumulation could contribute to an increase in ROS-mediated cardiolipin peroxidation. In support of this hypothesis, we demonstrate that E3 treatment is sufficient to prevent podocyte cytotoxicity in P sera-treated siABCA1 podocytes in vitro and albuminuria in Abca1-deficient diabetic mice. Both E3 treatment of ob/ob or pharmacological treatment of $\mathrm{db} / \mathrm{db}$ mice with an ABCA1 inducer are sufficient to reduce cardiolipin oxidation and prevent the progression of DKD. These data further validate our previously published findings (14) that ABCA1 deficiency contributes to podocyte injury and that ABCA1 may be a therapeutic target for the treatment of DKD. Additionally, the observations that total cardiolipin content is increased in ABCA1 deficiency and that upregulation of Abca1 as shown in vivo results in a downregulation of DHA-rich cardiolipin species (relevant in oxidant production, ref. 37) suggests that agents targeting cardiolipin oxidation specifically through fatty-acid side chain alterations may represent a new treatment for DKD and other chronic diseases and mitochondrial disorders where lipid dysregulation and mitochondrial dysfunction are prevalent. Several groups identified an indirect link between ABCA1 and mitochondrial function through the use of micro-RNA-based therapies $(51,52)$. Specifically, miR-33, a micro-RNA found to target sterol regulatory binding protein 2 (SREBP2), downregulates ABCA1 expression. Inhibition of miR-33 results in an increase of ABCA1 expression in pancreatic islet cells. Similarly, inhibition of
miR-33 with a selective anti-miR-33 resulted in upregulation of ABCA1 expression and function and was associated with a significant increase in mitochondrial respiration and ATP production in macrophages (51-53). Treatment with an inhibitor of miR-33 also improves atherosclerosis in mice. These data suggest an association among ABCA1, lipids, and mitochondrial function, supporting our finding that $\mathrm{ABCA1}$ deficiency can directly cause mitochondrial dysfunction. Fibroblasts from patients with ABCA1 deficiency (Tangier disease) are characterized by an accumulation of cardiolipin. Patient case studies demonstrated that patients with Tangier disease have lipid accumulation in the kidney $(15,54,55)$, further supporting the notion that ABCA1 deficiency contributes to lipid accumulation and particularly cardiolipin accumulation in mitochondria, as demonstrated in this study.

Proper cardiolipin synthesis and content are important for maintaining the integrity of the inner mitochondrial membrane and for the biogenesis and stability of OXPHOS complexes and supercomplexes $(24,26,56-59)$. In ABCA1-deficient podocytes, the observed increase in cardiolipin content is associated with the remodeling of the OXPHOS system. In particular, CI-containing supercomplexes accumulate in siABCA1 as compared with siCO podocytes. This does not appear to be a consequence of an overall induction in mitochondrial biogenesis, but rather of changes in the organization of the mitochondrial respiratory chain that favor the assembly or stability of higher ordered OXPHOS-complex structures. Indeed, the total amounts of OXPHOS individual respiratory complexes in mitochondria are not affected, with the exception of CI steady-state levels. This observation is consistent with the notion that the entire mitochondrial CI pool is known to be comprised into the respirasomes (60).

The functional role of mitochondrial supercomplexes has not been fully elucidated. It has been proposed that these structures could increase electron transfer efficiency from CI and so confer kinetic advantages to the system. Here, the decrease in CII but not CI substrate-driven respiration in permeabilized cells could be explained by the fact that CII-driven electrons flow through free $\mathrm{CIII}_{2}$ (which are less abundant in siABCA1 podocytes) and CIV complexes, whereas CI-driven electrons flow through the CI-CIII-CIV supercomplexes (which are more abundant in siABCA1 podocytes). Paradoxically, despite the increase in mitochondrial respirasome levels and CI activity, we observed a significant decrease in endogenous cell respiration in siABCA1 podocytes compared with siCO podocytes. Podocytes are high-energy-demanding cells, heavily relying on oxidative metabolism for ATP production (61). However, little is known regarding the type of energy substrate or substrates primarily used by these cells and their metabolic flexibility (62). We can speculate that if their primary energy source were NADHlinked substrates, the OXPHOS respiratory complex organization seen in siABCA1 podocytes should be optimal to support cellular respiration. On the contrary, if they would preferentially use FADHlinked substrates (e.g., succinate or fatty acids), that organization would be suboptimal to maximum substrate utilization. One would expect the OXPHOS respiratory complex organization to be flexible and dynamic, as has been proposed (63). However, it is possible that the significant excess of cardiolipin measured in the mitochondrial membranes could impede dynamics and therefore adaptations to changes in substrate availability. 
Furthermore, the amount of cardiolipin present at the inner-mitochondrial membrane plays an important role in cell death. Huang et al. demonstrated that cardiolipin-deficient HeLa cells have reduced cardiolipin peroxidation associated with resistance to apoptosis (64). Other groups reported that among all mitochondrial lipids, cardiolipin is preferentially oxidized during apoptosis and that oxidized cardiolipin has a lower affinity to cytochrome $c$, which allows the release of cytochrome $c$ from the inner mitochondrial membrane to start the cell death cascade $(25,42$, 65-68). These data support our findings where cardiolipin accumulation in an environment with high peroxidase activity, such as in diabetes, results in increased podocyte injury and worsened $\mathrm{DKD}$, whereas the stabilization of cardiolipin at the inner mitochondrial membrane together with the inhibition of cytochrome $c$ peroxidase (preventing cardiolipin oxidation) improves podocyte integrity and DKD outcomes $(38,69)$.

In summary, we identified a novel mechanism by which ABCA1 deficiency contributes to cardiolipin accumulation and mitochondrial dysfunction, leading to podocyte injury and to the progression of DKD. We also demonstrate that pharmacological induction of ABCA1 expression or inhibition of cardiolipin peroxidation may represent a novel therapeutic strategy for the treatment of patients with DKD and potentially for the treatment of other disorders in which altered glomerular lipid metabolism contributes to podocyte injury.

\section{Methods}

Study approval. All animal studies were approved by the IACUC at the University of Miami. All study protocols involving human samples were granted IRB exemption since the samples were collected under an IRB-approved research study for which informed consent was obtained and there was no access to identifiable information. Appropriate safeguards were in place to protect subject confidentiality and privacy. Additional information about Methods can be found in the Supplemental Material.

\section{Author contributions}

GMD conceived the project, performed the in vitro and in vivo experiments, analyzed the data, and wrote the manuscript. CEP performed the cholesterol efflux assays with patient sera. GWB helped design the cell-based assay utilizing patient sera. SKM and SB helped with the mass spectrometry analysis of lipids. AM, SKM, JM, JVS, XL, MG, JJK, and YH assisted with in vivo experiments. AS conducted the STZ injections and helped with some in vivo experiments. AJM designed experiments related to cholesterol determination and efflux. MK and VN performed microarray analysis utilizing patient sera provided by RGN. YI, RI, HHS, and SL performed TEM experiments. CM contributed to the development and characterization of the ABCA1 inducer used in this study. SM, FF, and AF conceived the project, designed and supervised the study, analyzed the data, and edited the manuscript. AF is the guarantor of this work and, as such, had full access to all the data in the study and takes responsibility for the integrity of the data and the accuracy of the data analysis.

\section{Acknowledgments}

AF is supported by NIH grants R01DK117599, R01DK104753, R01CA227493, U54DK083912, UM1DK100846, U01DK116101, and UL1TR000460 (Miami Clinical Translational Science Institute). RGN was supported by the Intramural Research Program of the National Institute of Diabetes and Digestive and Kidney Disease. GMD was supported by a predoctoral fellowship of the American Heart Association (16PRE30200010). FF is supported by a Scientist Development Grant of the American Heart Association (14SDG20040003). We thank Dr John Parks for the Abca1 $1^{\mathrm{f} / \mathrm{fl}}$ mice and Dr Laura Barisoni for her histological assessments of the $A b c a 1^{\mathrm{f} / / \mathrm{l}} \mathrm{ob} / \mathrm{ob}$ mouse model. We thank Kevin Johnson for the PAS stainings done in all mouse models and Dr Mayrin Correa for technical assistance in some of the in vitro experiments. We give a special thanks to the Katz family for continuous support.

Address correspondence to: Alessia Fornoni, Katz Family Division of Nephrology and Hypertension and Peggy and Harold Katz Family Drug Discovery Center, University of Miami, 1580 NW 10th Avenue, Miami, Florida 33136, USA. Phone: 305.243.7745; Email: afornoni@med.miami.edu. Or to: Flavia Fontanesi, Department of Biochemistry and Molecular Biology, University of Miami, Neuroscience Research Building \#103B, 1420 NW 9th Avenue, Miami, FL 33136 USA. Phone: 305.243.7215; Email: ffontanesi@ med.miami.edu.
1. US Renal Data System 2016 Annual Data Report: Epidemiology of Kidney Disease in the United States. Am J Kidney Dis. 2017;69(3S1):A4.

2. Patney V, Whaley-Connell A, Bakris G. Hypertension management in diabetic kidney disease. Diabetes Spectr. 2015;28(3):175-180.

3. de Borst MH, Navis G. Diabetes: Risks of strict glycaemic control in diabetic nephropathy. Nat Rev Nephrol. 2015;11(1):5-6.

4. Anders HJ, Huber TB, Isermann B, Schiffer M. CKD in diabetes: diabetic kidney disease versus nondiabetic kidney disease. Nat Rev Nephrol. 2018;14(6):361-377.

5. Pagtalunan ME, et al. Podocyte loss and progressive glomerular injury in type II diabetes. J Clin Invest. 1997;99(2):342-348.

6. Meyer TW, Bennett PH, Nelson RG. Podocyte number predicts long-term urinary albumin excretion in Pima Indians with Type II diabetes and microalbuminuria. Diabetologia. 1999;42(11):1341-1344.

7. Pavenstädt H, Kriz W, Kretzler M. Cell biology of the glomerular podocyte. Physiol Rev. 2003;83(1):253-307.

8. Herman-Edelstein M, Scherzer P, Tobar A, Levi M, Gafter U. Altered renal lipid metabolism and renal lipid accumulation in human diabetic nephropathy. J Lipid Res. 2014;55(3):561-572.

9. Merscher-Gomez S, et al. Cyclodextrin protects podocytes in diabetic kidney disease. Diabetes. 2013;62(11):3817-3827.

10. Wang Z, et al. Regulation of renal lipid metabolism, lipid accumulation, and glomerulosclerosis in FVBdb/db mice with type 2 diabetes. Diabetes. 2005;54(8):2328-2335.

11. Proctor G, Jiang T, Iwahashi M, Wang Z, Li J, Levi
M. Regulation of renal fatty acid and cholesterol metabolism, inflammation, and fibrosis in Akita and OVE26 mice with type 1 diabetes. Diabetes. 2006;55(9):2502-2509.

12. Wang N, Silver DL, Thiele C, Tall AR. ATP-binding cassette transporter A1 (ABCA1) functions as a cholesterol efflux regulatory protein. J Biol Chem. 2001;276(26):23742-23747.

13. Oram JF, Lawn RM. ABCA1. The gatekeeper for eliminating excess tissue cholesterol. J Lipid Res. 2001;42(8):1173-1179.

14. Pedigo CE, et al. Local TNF causes NFATc1dependent cholesterol-mediated podocyte injury. J Clin Invest. 2016;126(9):3336-3350.

15. Ferrans VJ, Fredrickson DS. The pathology of Tangier disease. A light and electron microscopic study. Am J Pathol. 1975;78(1):101-158.

16. Fobker M, Voss R, Reinecke H, Crone C, Assmann 
G, Walter M. Accumulation of cardiolipin and lysocardiolipin in fibroblasts from Tangier disease subjects. FEBS Lett. 2001;500(3):157-162.

17. Brooks-Wilson A, et al. Mutations in $\mathrm{ABC} 1$ in Tangier disease and familial high-density lipoprotein deficiency. Nat Genet.1999;22(4):336-345.

18. Bodzioch $\mathrm{M}$, et al. The gene encoding ATP-binding cassette transporter 1 is mutated in Tangier disease. Nat Genet. 1999;22(4):347-351.

19. Forbes JM, Thorburn DR. Mitochondrial dysfunction in diabetic kidney disease. Nat Rev Nephrol. 2018;14(5):291-312.

20. Kampe K, Sieber J, Orellana JM, Mundel P, Jehle AW. Susceptibility of podocytes to palmitic acid is regulated by fatty acid oxidation and inversely depends on acetyl-CoA carboxylases 1 and 2. Am JPhysiol Renal Physiol. 2014;306(4):F401-F409.

21. Sieber J, et al. Susceptibility of podocytes to palmitic acid is regulated by stearoyl-CoA desaturases 1 and 2. Am J Pathol. 2013;183(3):735-744.

22. Sharma K, et al. Metabolomics reveals signature of mitochondrial dysfunction in diabetic kidney disease. J Am Soc Nephrol. 2013;24(11):1901-1912.

23. Qi H, et al. Glomerular endothelial mitochondrial dysfunction is essential and characteristic of diabetic kidney disease susceptibility. Diabetes. 2017;66(3):763-778.

24. Paradies G, Paradies V, De Benedictis V, Ruggiero FM, Petrosillo G. Functional role of cardiolipin in mitochondrial bioenergetics. Biochim Biophys Acta. 2014;1837(4):408-417.

25. Szeto HH. First-in-class cardiolipin-protective compound as a therapeutic agent to restore mitochondrial bioenergetics. Br J Pharmacol. 2014;171(8):2029-2050.

26. Birk AV, Chao WM, Bracken C, Warren JD, Szeto $\mathrm{HH}$. Targeting mitochondrial cardiolipin and the cytochrome c/cardiolipin complex to promote electron transport and optimize mitochondrial ATP synthesis. Br JPharmacol. 2014;171(8):2017-2028.

27. Warner GJ, Stoudt G, Bamberger M, Johnson WJ, Rothblat GH. Cell toxicity induced by inhibition of acyl coenzyme A: cholesterol acyltransferase and accumulation of unesterified cholesterol. J Biol Chem. 1995;270(11):5772-5778.

28. Tabas I. Consequences of cellular cholesterol accumulation: basic concepts and physiological implications. JClin Invest. 2002;110(7):905-911.

29. Wang XX, et al. Diabetic nephropathy is accelerated by farnesoid $\mathrm{X}$ receptor deficiency and inhibited by farnesoid $\mathrm{X}$ receptor activation in a type 1 diabetes model. Diabetes. 2010;59(11):2916-2927.

30. Griparic L, van der Wel NN, Orozco IJ, Peters PJ, van der Bliek AM. Loss of the intermembrane space protein Mgm1/OPA1 induces swelling and localized constrictions along the lengths of mitochondria. JBiol Chem. 2004;279(18):18792-18798.

31. Czajka A, et al. Altered mitochondrial function, mitochondrial DNA and reduced metabolic flexibility in patients with diabetic nephropathy. EBioMedicine. 2015;2(6):499-512.

32. Kolavennu V, Zeng L, Peng H, Wang Y, Danesh FR. Targeting of RhoA/ROCK signaling ameliorates progression of diabetic nephropathy independent of glucose control. Diabetes. 2008;57(3):714-723.

33. Kang HM, et al. Defective fatty acid oxidation in renal tubular epithelial cells has a key role in kidney fibrosis development. Nat Med. 2015;21(1):37-46.

34. Beauchamp J, Kemmer C, Mohr P, Roever S, Wright M. Methods of identifying modulators of osbpl7 the use of such modulators for treatment of diseases associated with osbpl7. Patent WO2014180741A1. 2013.

35. Hong MY, et al. Fish oil increases mitochondrial phospholipid unsaturation, upregulating reactive oxygen species and apoptosis in rat colonocytes. Carcinogenesis. 2002;23(11):1919-1925.

36. Watkins SM, Carter LC, German JB. Docosahexaenoic acid accumulates in cardiolipin and enhances HT-29 cell oxidant production. JLipid Res. 1998;39(8):1583-1588.

37. Sullivan EM, et al. Docosahexaenoic acid lowers cardiac mitochondrial enzyme activity by replacing linoleic acid in the phospholipidome. J Biol Chem. 2018;293(2):466-483.

38. Birk AV, et al. The mitochondrial-targeted compound SS-31 re-energizes ischemic mitochondria by interacting with cardiolipin. J Am Soc Nephrol. 2013;24(8):1250-1261.

39. Hudkins KL, et al. BTBR Ob/Ob mutant mice model progressive diabetic nephropathy. JAm Soc Nephrol. 2010;21(9):1533-1542.

40. Tsun JG, Yung S, Chau MK, Shiu SW, Chan TM, Tan KC. Cellular cholesterol transport proteins in diabetic nephropathy. PLoS One. 2014;9(9):e105787.

41. Sakamoto Y, et al. Vancomycin induces reactive oxygen species-dependent apoptosis via mitochondrial cardiolipin peroxidation in renal tubular epithelial cells. Eur JPharmacol. 2017;800:48-56.

42. Yin H, Zhu M. Free radical oxidation of cardiolipin: chemical mechanisms, detection and implication in apoptosis, mitochondrial dysfunction and human diseases. Free Radic Res. 2012;46(8):959-974.

43. Murphy MP. Mitochondrial dysfunction indirectly elevates ROS production by the endoplasmic reticulum. Cell Metab. 2013;18(2):145-146.

44. Leadsham JE, et al. Loss of cytochrome c oxidase promotes RAS-dependent ROS production from the ER resident NADPH oxidase, Ynolp, in yeast Cell Metab. 2013;18(2):279-286.

45. Xiao L, et al. The mitochondria-targeted antioxidant MitoQ ameliorated tubular injury mediated by mitophagy in diabetic kidney disease via Nrf2/PINK1. Redox Biol. 2017;11:297-311.

46. Kitada M, Kanasaki K, Koya D. Clinical therapeutic strategies for early stage of diabetic kidney disease. World J Diabetes. 2014;5(3):342-356.

47. Susztak K, Raff AC, Schiffer M, Böttinger EP. Glucose-induced reactive oxygen species cause apoptosis of podocytes and podocyte depletion at the onset of diabetic nephropathy. Diabetes. 2006;55(1):225-233.

48. Zhang G, Darshi M, Sharma K. The Warburg effect in diabetic kidney disease. Semin Nephrol. 2018;38(2):111-120.

49. Zou HH, Yang PP, Huang TL, Zheng XX, Xu GS. PLK2 plays an essential role in high D-glucoseinduced apoptosis, ROS generation and inflammation in podocytes. Sci Rep. 2017;7(1):4261.

50. Galvan DL, et al. Real-time in vivo mitochondrial redox assessment confirms enhanced mitochondrial reactive oxygen species in diabetic nephropathy. Kidney Int. 2017;92(5):1282-1287.
51. Karunakaran D, et al. Macrophage mitochondrial energy status regulates cholesterol efflux and is enhanced by anti-mir33 in atherosclerosis. Circ Res. 2015;117(3):266-278.

52. Wijesekara N, et al. miR-33a modulates ABCA1 expression, cholesterol accumulation, and insulin secretion in pancreatic islets. Diabetes. 2012;61(3):653-658.

53. Horie T, et al. MicroRNA-33 regulates sterol regulatory element-binding protein 1 expression in mice. Nat Commun. 2013;4:2883.

54. Bale PM, Clifton-Bligh P, Benjamin BN, Whyte HM. Pathology of Tangier disease. JClin Pathol. 1971;24(7):609-616.

55. Clifton-Bligh P, Nestel PJ, Whyte HM. Tangier disease. Report of a case and studies of lipid metabolism. N EnglJ Med. 1972;286(11):567-571.

56. Schlame M, Ren M. The role of cardiolipin in the structural organization of mitochondrial membranes. Biochim Biophys Acta. 2009;1788(10):2080-2083.

57. Bazán S, Mileykovskaya E, Mallampalli VK, Heacock P, Sparagna GC, Dowhan W. Cardiolipin-dependent reconstitution of respiratory supercomplexes from purified Saccharomyces cerevisiae complexes III and IV. J Biol Chem. 2013;288(1):401-411.

58. Fry M, Green DE. Cardiolipin requirement for electron transfer in complex I and III of the mitochondrial respiratory chain. J Biol Chem. 1981;256(4):1874-1880.

59. Claypool SM, Koehler CM. The complexity of cardiolipin in health and disease. Trends Biochem Sci. 2012;37(1):32-41.

60. Barrientos A, Ugalde C. I function, therefore I am: overcoming skepticism about mitochondria supercomplexes. Cell Metab. 2013;18(2):147-149.

61. Imasawa $T$, et al. High glucose repatterns human podocyte energy metabolism during differentiation and diabetic nephropathy. FASEB J. 2017;31(1):294-307.

62. Imasawa $T$, Rossignol R. Podocyte energy metabolism and glomerular diseases. Int J Biochem Cell Biol. 2013;45(9):2109-2118.

63. Acín-Pérez R, Fernández-Silva P, Peleato ML, Pérez-Martos A, Enriquez JA. Respiratory active mitochondrial supercomplexes. Mol Cell. 2008;32(4):529-539.

64. Huang $Z$, et al. Cardiolipin deficiency leads to decreased cardiolipin peroxidation and increased resistance of cells to apoptosis. Free Radic Biol Med. 2008;44(11):1935-1944.

65. Basova LV, et al. Cardiolipin switch in mitochondria: shutting off the reduction of cytochrome $\mathrm{c}$ and turning on the peroxidase activity. Biochemistry. 2007;46(11):3423-3434.

66. Montero J, et al. Cholesterol and peroxidized cardiolipin in mitochondrial membrane properties, permeabilization and cell death. Biochim Biophys Acta. 2010;1797(6-7):1217-1224.

67. Kagan VE, et al. Cytochrome c acts as a cardiolipin oxygenase required for release of proapoptotic factors. Nat Chem Biol. 2005;1(4):223-232.

68. Jiang X, Wang X. Cytochrome C-mediated apoptosis. Annu Rev Biochem. 2004;73:87-106.

69. Belikova NA, et al. Peroxidase activity and structural transitions of cytochrome $\mathrm{c}$ bound to cardiolipin-containing membranes. Biochemistry. 2006;45(15):4998-5009. 